\title{
Synthesis of a New Cerebroside from a Chondropsis sp. Sponge ${ }^{1,2)}$
}

\author{
Masanori Honda, Yoshitaka Ueda, Shigeo Sugryama, and Tetsuya Komori* \\ Faculty of Pharmaceutical Sciences, Kyushu University, Maidashi 3-1-1, Higashi-ku, Fukuoka 812, Japan. Received October 29, 1990
}

\begin{abstract}
A cerebroside, 1- $O$ - $(\beta$-D-galactopyranosyloxy $)-(2 S, 3 S, 4 R, 6 E)-2-[(R)-2$-hydroxytetracosanoylamino]-17-methyl6-octadecene-3,4-diol (2), was asymmetrically synthesized from isobutyraldehyde. On the basis of a comparison of the physical data, the absolute structure of a new cerebroside $1 \mathrm{~b}$ from a Chondropsis sp. sponge is thought to be the same as that of 2.
\end{abstract}

Keywords glycosphingolipid; asymmetric synthesis; absolute stereochemistry; phytosphingosine; galactosyl ceramide; hystidine decarboxylase; hypotensive activity; $(R)$-benzyl-2,3-epoxypropyl ether; sponge; Chondropsis sp.

Recently, a mixture of new galactosyl ceramides (1a and 1b) was separated from a sea sponge, Chondropsis sp., and was shown to inhibit hystidine decarboxylase as well as to have hypotensive activity in anesthetized rats. ${ }^{2)}$ The mixture was characterized by acid hydrolysis, ozonolysis, and gas chromatography-mass spectrometry (GC-MS) analysis and found to be composed of D-galactose, $\alpha$-hydroxytetracosanoic acid, and $\mathrm{C}_{17}-$ and $\mathrm{C}_{18}$-phytosphingosines bearing a trans-double bond and an iso-type terminal in the long chain, as illustrated in Chart 1 . However, neither the determination of the absolute stereochemistry nor the isolation of the pure cerebrosides $1 \mathrm{a}$ and $1 \mathrm{~b}$ was achieved.

As a part of our studies on glycosphingolipids from starfishes, we were primarily interested in the absolute structure and biological activities of the new cerebrosides $1 \mathbf{a}$ and $\mathbf{1 b}$. While the syntheses of many kinds of ceramides and cerebrosides have been achieved, ${ }^{3)}$ there seems to be no documentation of the synthesis of a phytosphingosinetype cerebroside possessing a hydroxy fatty acid, except for one example by our group. ${ }^{4)}$ For the synthesis of the new cerebrosides $1 \mathrm{a}$ and $1 \mathrm{~b}$, we focused on the synthesis of the ceramide, $(2 S, 3 S, 4 R, 6 E)-2-[(R)-2$-hydroxytetracosanoylamino]-17-methyl-6-octadecene-1,3,4-triol (2), which contains the most common configurations among naturally occurring phytosphingosines and $\alpha$-hydroxy fatty acids. The design of the synthesis of $\mathbf{2}$ is retrosynthetically outlined in Chart 1 . The chiralities on the phytosphingosine were successively introduced into the $\mathrm{C}-4, \mathrm{C}-3$, and $\mathrm{C}-2$ positions by utilizing the chiral synthon $(R)$-benzyl-2,3-epoxypropyl ether (7), asymmetric epoxidation, and amination with a regioselective epoxide opening, leading to 5. $(R)-2$ Hydroxytetracosanoic acid (4) has been prepared by our group. ${ }^{4 a}$ The acid 4 and the phytosphingosine 5 were converted into the cerebroside 2 after glycosidation of the ceramide 3 with D-galactose.

Isobutyraldehyde (9) was used for the construction of the iso-type terminal of a phytosphingosine, $(2 S, 3 S, 4 R, 6 E)$ 2-amino-17-methyl-6-octadecene-1,3,4-triol (5). A Wittig reaction of 9 with $\alpha, \alpha$-dibromomethylenetriphenylphosphorane $^{5)}$ gave an olefinic dibromide 10 in an $88 \%$ yield. The dibromide 10 was treated first with $n$-butyllithium and then bromooctane to afford an alkyne 11 in a $70 \%$ yield. Hexamethylphosphoric triamide (HMPA) and three equivalents of $n$-butyllithium are necessary for this alkylation. The migration of the internal triple bond of 11 with potassium aminopropylamide (KAPA) ${ }^{6}$ ) resulted in the alkyne 8 , of which the terminals were an iso-type moiety and a triple bond. The alkyne 8 was treated with $n$ butyllithium and then $(R)$-benzyl-2,3-epoxypropyl ether $(7)^{7)}$ to produce a chiral alcohol, which was protected as the methoxymethoxy (MOM) ether (12) with chloromethyl methyl ether (MOMCl) and $N, N$-diisopropylethylamine in a $64 \%$ yield from 8. Debenzylation and the reduction of the MOM ether 12 with lithium in ethylamine and tert-

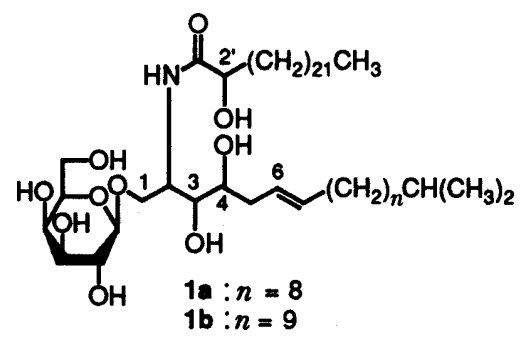

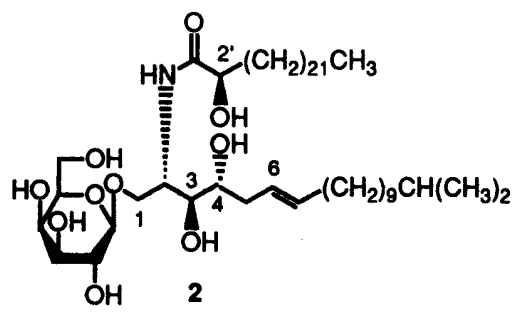

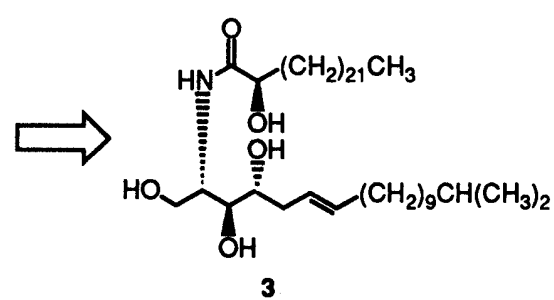<smiles>CCC[C@H](O)C(=O)O</smiles><smiles>CCCC</smiles><smiles>CO[C@H](C=C(C)CC(C)C)[C@H]1CO1</smiles>
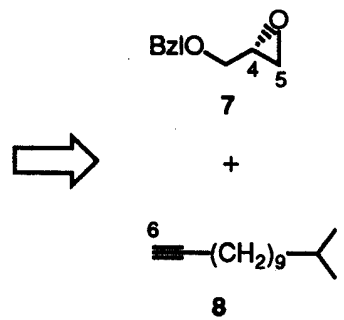

Chart 1

(C) 1991 Pharmaceutical Society of Japan 


$$
\begin{aligned}
& \underbrace{}_{9} \mathrm{CHO} \frac{\mathrm{PPh}_{3}, \mathrm{CBr}_{4},}{\mathrm{CH}_{2} \mathrm{Cl}_{2}, 88 \%} \underbrace{\gamma_{\mathrm{Br}}}_{10} \mathrm{Br} \frac{n-\mathrm{BuLi}, \mathrm{THF}_{1}}{\underset{70 \%}{\mathrm{C}_{8} \mathrm{H}_{17} \mathrm{Br}, \mathrm{HMPA},}}
\end{aligned}
$$

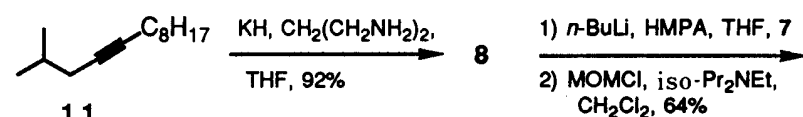

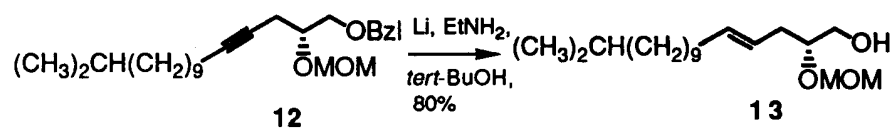

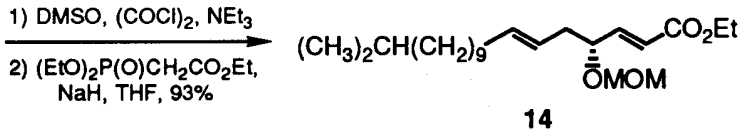$$
\underset{93 \%}{\stackrel{\mathrm{DIBAH}, \mathrm{Et}_{2} \mathrm{O},}{\longrightarrow}}\left(\mathrm{CH}_{3}\right)_{2} \mathrm{CH}\left(\mathrm{CH}_{2}\right)_{9} \overbrace{15}^{\mathrm{O} M O M} \mathrm{OH}
$$

Chart 2

butanol gave an alcohol (13) bearing a trans-double bond in an $80 \%$ yield. Swern oxidation ${ }^{8)}$ of 13 followed by Horner-Emmons reaction afforded a trans- $\alpha, \beta$-unsaturated ester 14 in a $93 \%$ yield. The ester 14 was reduced with diisobutylaluminum hydride (DIBAH) to give the $(4 R)$ allylic alcohol 15 in a $93 \%$ yield.

Asymmetric epoxidation ${ }^{9)}$ of 15, using (-)-diisopropyl tartrate (DIPT) as a chiral auxiliary, gave a mixture of the $(2 R, 3 R, 4 R)$-epoxy alcohol 6 and the $(2 S, 3 S, 4 R)$-epoxy alcohol 16 in a ratio of $3: 1 .^{10)}$ The formation of the diastereomeric epoxy alcohols was confirmed by a comparison of the epoxy alcohol prepared by epoxidation of 15 with vanadyl acetylacetonate $\left[\mathrm{VO}(\mathrm{acac})_{2}\right]$ and tert-butyl hydroperoxide (TBHP). ${ }^{11)}$ The configurations of 6 and 16 were determined by examination of the phytosphingosine tetraacetates 23 and 26, as described later. The mixture of 6 and 16 was converted into the urethanes 17 and 18, which were subjected to an intramolecular base-catalyzed epoxide opening with sodium hydride in tetrahydrofuran (THF) ${ }^{12}$ ) to give $N$-benzyloxazolidinones 19 and 20 in 63 and $23 \%$ yields from 6 and 16, respectively, after separation by silica gel chromatography.

Debenzylation of the $N$-benzyloxazolidinone 19 afforded an oxazoline 21 which was subjected to alkaline hydrolysis and cleavage of the MOM protective group to produce the phytosphingosine 5. Acetylation of 5 to 23 and subsequent hydrogenation gave the phytosphingosine tetraacetate 25 . A comparison of the ${ }^{1} \mathrm{H}$ - and ${ }^{13} \mathrm{C}$-nuclear magnetic resonance (NMR) data of 25 with those of authentic $(2 S, 3 S, 4 R)$ phytosphingosine tetraacetate given in the literature ${ }^{4 b}$ ) revealed that the absolute structure of phytosphingosine 5 was $(2 S, 3 S, 4 R)$-2-amino-17-methyl-6-octadecene-1,3,4triol. The isomeric $N$-benzyloxazolidinone 20 was also converted sequentially into 22,24 , and 26 in a similar manner to that described above. The configurations of 24 were $2 R, 3 R$, and $4 R$ on the basis of a comparison of the ${ }^{1} \mathrm{H}$ - and ${ }^{13} \mathrm{C}-\mathrm{NMR}$ data on 26 with those of authentic phytosphingosine tetraacetate, $(2 R, 3 R, 4 R)$-2-acetamido1,3,4-triacetoxyhexadecane. ${ }^{4 b)}$

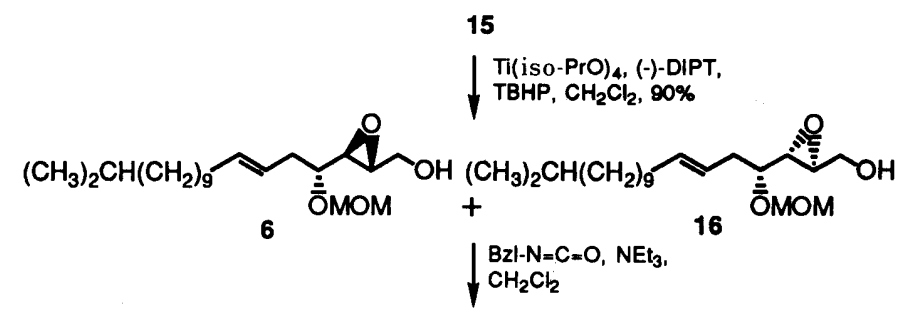

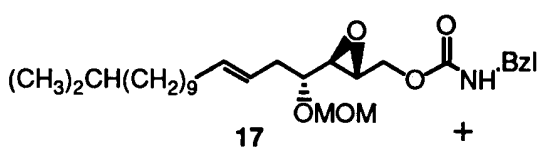<smiles>CCCCCC=CC[C@H]1OC1(CO)COC(=O)NCC</smiles>

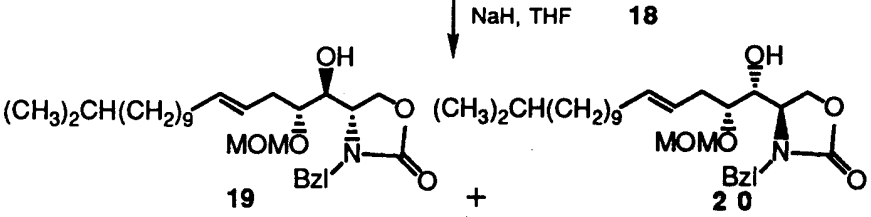

(63\% from a mixture of 6 and 16 )

(23\% from a mixture of 6 and 16 )

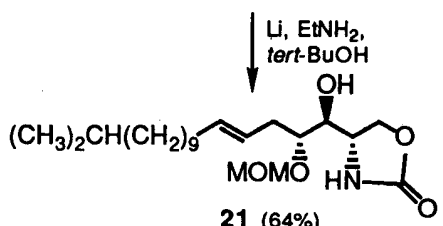

$21(64 \%)$

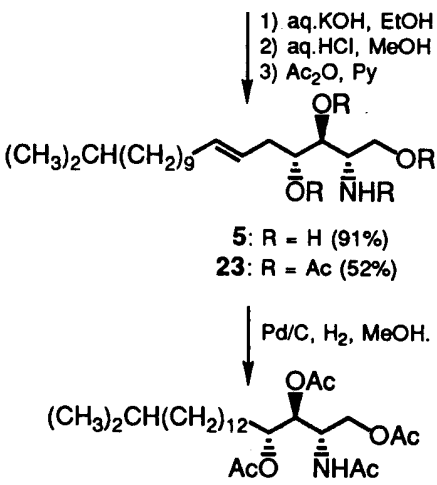

$25(59 \%)$<smiles>CC(C)CCCCC[C@@H](O)[C@@H](O)[C@H](N)CO</smiles>
$22(54 \%)$

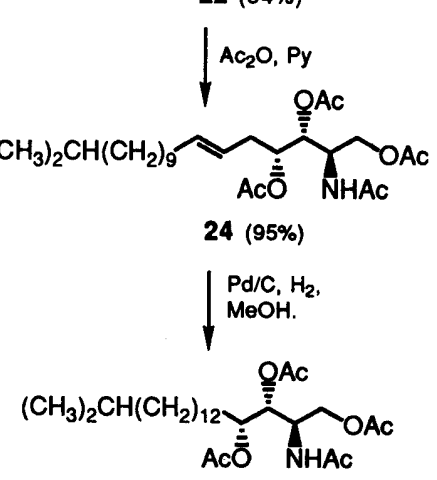

$26(60 \%)$
Chart 3

The amidation of 5 and ( $R$ )-2-(methoxymethoxy)tetracosanoic acid (27) was carried out with dicyclohexylcarbodiimide (DCC) and 1-hydroxybenzotriazole (HOBT) in $\mathrm{THF}^{13)}$ to give 28 in a $76 \%$ yield. The MOM protective group of 28 was removed with 1,2-ethanedithiol and boron trifluoride etherate ${ }^{14)}$ to give the ceramide 3 in a $76 \%$ yield. At this point, we could introduce all chiralities, the trans-double bond, and the iso-type terminal of the ceramide, requisite for synthesis of the cerebroside 2 . The secondary hydroxy groups of $\mathbf{3}$ were protected as benzoyl esters (except for the primary hydroxy group) by successive tritylation, benzoylation, and detritylation to afford 31 in an $84 \%$ yield. Glycosidation of 31 and a D-galactosyl imidate 32 in the presence of boron trifluoride etherate and $4 \AA$ molecular sieves in dichloromethane ${ }^{15)}$ gave 33 together with 34 , which was also formed in the glycosidation of a pentaacetylglucosyl imidate. ${ }^{3 b, 4 b)}$ Finally, deacylation of 33 with a methanolic potassium carbonate solution gave the D-galactosyl ceramide 2. All of the NMR data of 2 


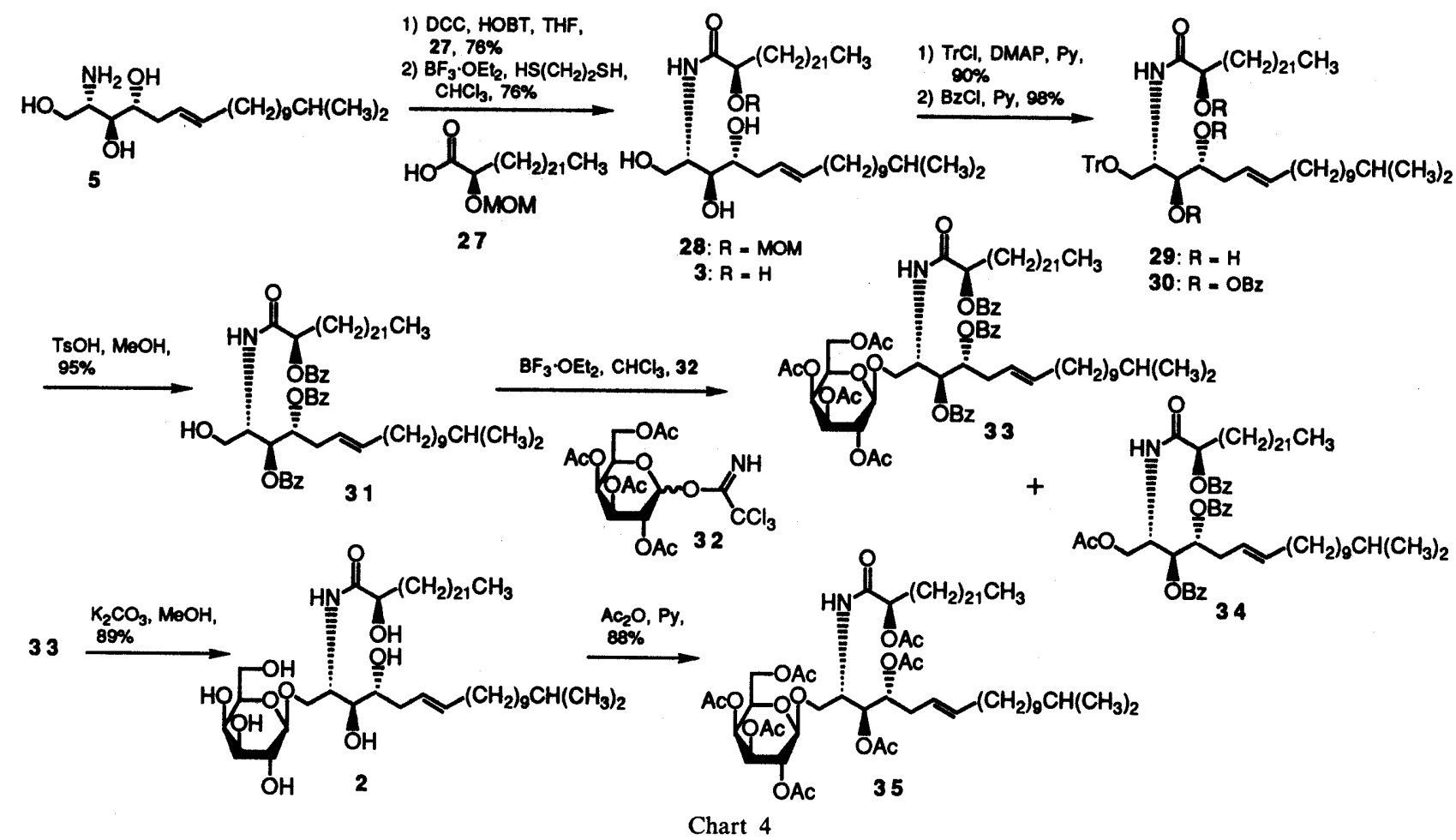

were in good agreement with those of the mixture of $1 \mathrm{a}$ and 1b. ${ }^{2)}$ Consequently, the first asymmetric synthesis of the new cerebroside $1 \mathrm{~b}$ with hystidine decarboxylase-inhibitory activity has been accomplished from isobutyraldehyde (9) in 22 steps. The cerebroside 2 was converted into a heptaacatate 35 in the usual manner. The ${ }^{1} \mathrm{H}$ - and ${ }^{13} \mathrm{C}$ NMR spectra of 35 were also in good agreement with those of the mixture of acetyl derivatives of $1 \mathrm{a}$ and $\mathbf{1 b}{ }^{2)}$

On the basis of the above results, the absolute structure of $1 \mathrm{~b}$ is thought to be $1-O$-( $\beta$-D-galactopyranosyloxy)$(2 S, 3 S, 4 R, 6 E)-2-[(R)-2$-hydroxytetracosanoylamino $]-17-$ methyl-6-octadecene-3,4-diol (2) and the other component of the mixture, 1a, which has the same absolute stereochemistry is considered to consist of a nor-phytosphingosine residue with the same hydroxytetracosanoyl group.

\section{Experimental}

Melting points were determined with a Yanaco micro melting point apparatus (MP-3). Optical rotations: Jasco DIP-360 polarimeter. Infrared (IR) spectra: Jasco IR-810 spectrometer. ${ }^{1} \mathrm{H}-\mathrm{NMR}$ spectra: $90 \mathrm{MHz}$, JEOL FX-90Q spectrometer; $270 \mathrm{MHz}$, JEOL GX-270 spectrometer; $400 \mathrm{MHz}$, JEOL GX-400 spectrometer. ${ }^{13} \mathrm{C}-\mathrm{NMR}$ spectra: $67.8 \mathrm{MHz}$, JEOL GX-270 spectrometer; $100 \mathrm{MHz}$, JEOL GX-400 spectrometer. NMR spectra were obtained by using $\mathrm{CDCl}_{3}, \mathrm{C}_{6} \mathrm{D}_{6}$, or pyridine- $d_{5}\left(\mathrm{C}_{5} \mathrm{D}_{5} \mathrm{~N}\right)$ as the solvent and tetramethylsilane as an internal standard. Field desorption mass spectra (FD-MS) and fast atom bombardment MS (FAB-MS): JEOL JMS-DX300/JMA-3500 data system. Electron impact MS (EI-MS) and high-resolution EI-MS: JEOL JMS-DX300/JMA-3100 data system. Silica gel column chromatography: Kieselgel 60 (70-230 mesh, No. 7734, Merck) if not otherwise specified, or Silica gel BW-300 (200-400 mesh, Fuji Davison Co., Ltd.). All solvents were distilled before use. Anhydrous THF and ether were distilled from sodium and benzophenone. Other anhydrous solvents were distilled from calcium hydride and stored over activated $4-\AA$ molecular sieve pellets. Reactions were carried out under argon, as required.

1,1-Dibromo-4-methyl-1-pentene (10) Carbon tetrabromide (12.4 g, $37.4 \mathrm{mmol})$ was added at $0^{\circ} \mathrm{C}$ to a solution of triphenylphosphine $(19.6 \mathrm{~g}$, $74.6 \mathrm{mmol})$ in $\mathrm{CH}_{2} \mathrm{Cl}_{2}(37 \mathrm{ml})$. The reaction mixture was stirred at room temperature for $1 \mathrm{~h}$ and isobutyraldehyde $9(2.0 \mathrm{ml}, 18.7 \mathrm{mmol})$ was added at $0{ }^{\circ} \mathrm{C}$ to the vigorously stirred mixture. Stirring was continued at room

temperature for $1 \mathrm{~h}$, then the mixture was diluted with hexane $(400 \mathrm{ml})$ and the resulting precipitate was filtered off through a cotton plug. The filtrate was concentrated under atmospheric pressure and distilled to give $10(3.98 \mathrm{~g}, 16.4 \mathrm{mmol}, 88 \%)$ as a colorless oil, bp $96^{\circ} \mathrm{C}$ (46 Torr). IR (neat): $2950(\mathrm{C}-\mathrm{H}), 1615(\mathrm{C}=\mathrm{C}), 1383$ and $1365\left[\mathrm{CH}-\left(\mathrm{CH}_{3}\right)_{2}\right] \mathrm{cm}^{-1}$. ${ }^{1} \mathrm{H}-\mathrm{NMR}\left(90 \mathrm{MHz}, \mathrm{CDCl}_{3}\right) \delta: 6.40(1 \mathrm{H}, \mathrm{t}, J=7.1 \mathrm{~Hz}, 2-\mathrm{H}), 2.00(2 \mathrm{H}, \mathrm{m}$, $3-\mathrm{H}), 1.73(1 \mathrm{H}, \mathrm{m}, 4-\mathrm{H}), 0.93\left(6 \mathrm{H}, \mathrm{d}, J=6.3 \mathrm{~Hz}, 5-\mathrm{H}\right.$ and $\left.\mathrm{CHCH}_{3}\right)$. Anal. Calcd for $\mathrm{C}_{6} \mathrm{H}_{10} \mathrm{Br}_{2}: \mathrm{C}, 29.79 ; \mathrm{H}, 4.17$. Found: $\mathrm{C}, 29.63 ; \mathrm{H}, 3.97$.

2-Methyl-4-tridecyne (11) $n$-Butyllithium $(73.2 \mathrm{ml}, 110 \mathrm{mmol}, 1.5 \mathrm{M}$ in hexane) was added at $-78^{\circ} \mathrm{C}$ to a solution of $10(8.57 \mathrm{~g}, 35.4 \mathrm{mmol})$ in THF $(71 \mathrm{ml})$. The reaction mixture was allowed to warm to room temperature over $1.5 \mathrm{~h}$ and then cooled to $-78^{\circ} \mathrm{C}$. A solution of 1-bromononane $(6.44 \mathrm{ml}, 37.2 \mathrm{mmol})$ in HMPA $(71 \mathrm{ml})$ was added at $-78^{\circ} \mathrm{C}$ to the mixture. After being stirred at $-78^{\circ} \mathrm{C}$ for $30 \mathrm{~min}$, the mixture was allowed to warm to room temperature over $1.5 \mathrm{~h}$, diluted with hexane, and washed with water. The aqueous layer was extracted with hexane. The combined hexane layer was dried $\left(\mathrm{Na}_{2} \mathrm{SO}_{4}\right)$, filtered, and concentrated in vacuo. The residue was distilled to give $11(4.85 \mathrm{~g}, 70 \%$ yield) as a colorless oil, bp $125^{\circ} \mathrm{C}$ (17 Torr). IR (neat): 2955 (C-H), 2930, 2850, 1383 and $1365\left[\mathrm{CH}-\left(\mathrm{CH}_{3}\right)_{2}\right] \mathrm{cm}^{-1},{ }^{1} \mathrm{H}-\mathrm{NMR}\left(90 \mathrm{MHz}, \mathrm{CDCl}_{3}\right) \delta$ : $2.08(4 \mathrm{H}, \mathrm{m}, 3-\mathrm{H}$ and $6-\mathrm{H}), 1.38(12 \mathrm{H}, \mathrm{m}), 0.96(6 \mathrm{H}, \mathrm{d}, J=6.4 \mathrm{~Hz}, 1-\mathrm{H}$ and $\left.\mathrm{CHCH}_{3}\right)$. EI-MS $m / z(\%): 194\left(\mathrm{M}^{+}, 2\right), 151\left(\mathrm{M}^{+}-\mathrm{C}_{3} \mathrm{H}_{7}, 5\right), 81$ $\left(\mathrm{M}^{+}-\mathrm{C}_{8} \mathrm{H}_{17}, 100\right)$. Anal. Calcd for $\mathrm{C}_{14} \mathrm{H}_{26}: \mathrm{C}, 86.51 ; \mathrm{H}, 13.48$. Found: C, $86.55 ; \mathrm{H}, 13.48$.

12-Methyl-1-tridecyne (8) 1,3-Diaminopropane $(31 \mathrm{ml})$ was added to KH $(2.24 \mathrm{~g}, 55.9 \mathrm{mmol} ; 20-25 \%$ wt. KH dispersion in mineral oil was washed 3 times with hexane and dried) under stirring and cooling in an ice bath. The reaction mixture was stirred at room temperature for $1 \mathrm{~h}$ and $11(5.97 \mathrm{~g}, 30.7 \mathrm{mmol})$ was added dropwise to the vigorously stirred mixture. Stirring was continued at room temperature for $5 \mathrm{~min}$, then the reaction mixture was quenched with water and extracted with hexane. The extracts were washed consecutively with water, $10 \%$ aqueous $\mathrm{HCl}$, and water, dried $\left(\mathrm{Na}_{2} \mathrm{SO}_{4}\right)$, filtered, and concentrated under atmospheric pressure. The residue was distilled to give $8(5.50 \mathrm{~g}, 92 \%$ yield) as a colorless oil, bp $85^{\circ} \mathrm{C}$ (1 Torr). IR (neat): 3315 ( $\left.\equiv \mathrm{C}-\mathrm{H}\right), 2955$ (C-H), 2930, 2855, $2120(\mathrm{C} \equiv \mathrm{C}), 1383$ and $1365\left[\mathrm{CH}-\left(\mathrm{CH}_{3}\right)_{2}\right] \mathrm{cm}^{-1} \cdot{ }^{1} \mathrm{H}-\mathrm{NMR}$ $\left(270 \mathrm{MHz}, \mathrm{CDCl}_{3}\right) \delta: 2.18(2 \mathrm{H}, \mathrm{dd}, J=7.0,2.6 \mathrm{~Hz}, 3-\mathrm{H}), 1.93(1 \mathrm{H}, \mathrm{t}, J=$ $2.6,1-\mathrm{H}), 1.27(10 \mathrm{H}, \mathrm{m}), 0.86\left(6 \mathrm{H}, \mathrm{d}, J=6.6 \mathrm{~Hz}, 13-\mathrm{H}\right.$ and $\left.\mathrm{CHCH}_{3}\right)$. EI-MS $m / z(\%): 194\left(\mathbf{M}^{+}, 1\right), 179\left(\mathbf{M}^{+}-\mathrm{CH}_{3}, 3\right), 151\left(\mathrm{M}^{+}-\mathrm{C}_{3} \mathrm{H}_{7}, 5\right)$, $109\left(\mathrm{M}^{+}-\mathrm{C}_{6} \mathrm{H}_{13}, 100\right)$. Anal. Calcd for $\mathrm{C}_{14} \mathrm{H}_{26}: \mathrm{C}, 86.51 ; \mathrm{H}, 13.48$. Found: $\mathrm{C}, 86.48 ; \mathrm{H}, 13.30$.

(2R)-1-Benzyloxy-2-(methoxymethoxy)-15-methyl-4-hexadecyne (12) $n$-Butyllithium $(5.37 \mathrm{ml}, 8.06 \mathrm{mmol}, 1.5 \mathrm{M}$ hexane solution) was added at $-25^{\circ} \mathrm{C}$ to a solution of $8(2.0 \mathrm{ml}, 8.06 \mathrm{mmol})$ in THF $(8 \mathrm{ml})$ and the 
mixture was allowed to warm to room temperature over $10 \mathrm{~min}$. The epoxide $7(1.48 \mathrm{ml}, 8.07 \mathrm{mmol})$ and HMPA $(16 \mathrm{ml})$ were added at $-25^{\circ} \mathrm{C}$ to the mixture. After being stirred at room temperature for $30 \mathrm{~min}$, the reaction mixture was diluted with hexane- $\mathrm{Et}_{2} \mathrm{O}(2: 1)$, washed with water, dried $\left(\mathrm{Na}_{2} \mathrm{SO}_{4}\right)$, filtered, and concentrated in vacuo. The residue was chromatographed on silica gel (hexane-AcOEt, $8: 2$ ) to give a mixture $(2.55 \mathrm{~g})$ of the desired alcohol and a trace of unreacted 7 . Chloromethyl methyl ether $(812 \mu \mathrm{l}, 10.7 \mathrm{mmol})$ and $N, N$-diisopropylethylamine $(2.11 \mathrm{ml}$ $12.1 \mathrm{mmol})$ were added to the mixture $(2.55 \mathrm{~g})$. After being stirred at room temperature for $20 \mathrm{~h}$, the reaction mixture was concentrated in vacuo and chromatographed on silica gel (hexane-AcOEt, $85: 15)$ to give $12(2.16 \mathrm{~g}$ $64 \%$ yield from 8$)$ as a colorless oil. $[\alpha]_{\mathrm{D}}^{26}-5.68^{\circ}\left(c=2.66, \mathrm{CHCl}_{3}\right)$. IR (neat): $2920(\mathrm{C}-\mathrm{H}), 2930,2855,735$ and $790(\mathrm{Ar}-\mathrm{H}) \mathrm{cm}^{-1} \cdot{ }^{1} \mathrm{H}-\mathrm{NMR}$ $\left(90 \mathrm{MHz}, \mathrm{CDCl}_{3}\right) \delta: 7.32(5 \mathrm{H}, \mathrm{s}$, aromatic $\mathrm{H}), 4.74\left(2 \mathrm{H}, \mathrm{s}, \mathrm{OCH}_{2} \mathrm{O}\right), 4.57$ $(2 \mathrm{H}, \mathrm{s}$, benzylic $\mathrm{H}), 3.85(1 \mathrm{H}, \mathrm{m}, 2-\mathrm{H}), 3.62(2 \mathrm{H}, \mathrm{dd}, J=4.9,1.1 \mathrm{~Hz}, 1-\mathrm{H})$ $3.38\left(3 \mathrm{H}, \mathrm{s}, \mathrm{OCH}_{3}\right), 2.48(2 \mathrm{H}, \mathrm{m}, 3-\mathrm{H}), 2.11(2 \mathrm{H}, \mathrm{br} \mathrm{s}, 6-\mathrm{H}), 1.25(\mathrm{~m}), 0.86$ $\left(6 \mathrm{H}, \mathrm{d}, J=6.1 \mathrm{~Hz}, 16-\mathrm{H}\right.$ and $\left.\mathrm{CHCH}_{3}\right)$. EI-MS $m / z(\%): 402\left(\mathrm{M}^{+}, 4\right), 357$ $\left(\mathrm{M}^{+}-\mathrm{CH}_{2} \mathrm{OCH}_{3}, 93\right), 207\left(\mathrm{M}^{+}-\mathrm{C}_{11} \mathrm{H}_{15} \mathrm{O}_{3}, 100\right)$. Anal. Calcd for $\mathrm{C}_{26} \mathrm{H}_{42} \mathrm{O}_{3}$ : C, 77.56; $\mathrm{H}, 10.51$. Found: $\mathrm{C}, 77.32 ; \mathrm{H}, 10.35$.

(2R)-2-(Methoxymethoxy)-15-methyl-4-hexadecen-1-ol (13) Liquid $\mathrm{EtNH}_{2}(50 \mathrm{ml})$ was added at $0^{\circ} \mathrm{C}$ to a solution of $12(2.05 \mathrm{~g}, 5.09 \mathrm{mmol})$ in tert-BuOH $(10 \mathrm{ml})$, then several pieces of lithium $(300 \mathrm{mg})$ were added at $-20^{\circ} \mathrm{C}$. The blue-colored mixture was stirred at $-20^{\circ} \mathrm{C}$ for $10 \mathrm{~min}$ and quenched with ammonium chloride. The unreacted lithium was removed, then the mixture was concentrated to half the original volume diluted with ether, washed with water, dried $\left(\mathrm{Na}_{2} \mathrm{SO}_{4}\right)$, filtered, and concentrated in vacuo. The residue was chromatographed on silica gel (hexane-AcOEt, $7: 3)$ to give $13(1.28 \mathrm{~g}, 80 \% \text { yield) as a colorless oil. [ } \alpha]_{\mathrm{D}}^{26}$ $-20.0^{\circ}\left(c=2.72, \mathrm{CHCl}_{3}\right)$. IR (neat): $3440(\mathrm{O}-\mathrm{H}), 2920(\mathrm{C}-\mathrm{H}), 2855,1381$ and $1362\left[\mathrm{CH}-\left(\mathrm{CH}_{3}\right)_{2}\right] \mathrm{cm}^{-1}$. ${ }^{1} \mathrm{H}-\mathrm{NMR}\left(90 \mathrm{MHz}, \mathrm{CDCl}_{3}\right) \delta: 4.71(2 \mathrm{H}$, $\left.\mathrm{s}, \mathrm{OCH}_{2} \mathrm{O}\right), 3.54(3 \mathrm{H}, \mathrm{m}, 1-\mathrm{H}$ and $2-\mathrm{H}), 3.42\left(3 \mathrm{H}, \mathrm{s}, \mathrm{OCH}_{3}\right), 2.82(1 \mathrm{H}$, $\mathrm{m}, \mathrm{OH}), 2.22(2 \mathrm{H}, \mathrm{m}, 3-\mathrm{H}), 1.99(2 \mathrm{H}, \mathrm{m}, 6-\mathrm{H}), 1.25(\mathrm{~m}), 0.86(6 \mathrm{H}, \mathrm{d}$ $J=6.1 \mathrm{~Hz}, 16-\mathrm{H}$ and $\left.\mathrm{CHCH}_{3}\right)$. FD-MS $m / z(\%): 315\left(\mathrm{M}^{+}+\mathrm{H}, 100\right), 105$ (50). Anal. Calcd for $\mathrm{C}_{19} \mathrm{H}_{38} \mathrm{O}_{3}$ : C, 72.56; H, 12.18. Found: C, 72.27; $\mathrm{H}$, 12.23 .

Ethyl (2E,4R,6E)-4-(Methoxymethoxy)-17-methyl-2,6-octadecadien-1ate (14) Dimethyl sulfoxide (DMSO) $(549 \mu \mathrm{l}, 7.74 \mathrm{mmol})$ was added at $-60^{\circ} \mathrm{C}$ to a solution of oxalyl chloride $(506 \mu \mathrm{l}, 5.80 \mathrm{mmol})$ in $\mathrm{CH}_{2} \mathrm{Cl}_{2}$ $(9.67 \mathrm{ml})$. The mixture was stirred at $-60^{\circ} \mathrm{C}$ for $2 \mathrm{~min}$ and a solution of $13(1.21 \mathrm{~g}, 3.87 \mathrm{mmol})$ in $\mathrm{CH}_{2} \mathrm{Cl}_{2}(3.87 \mathrm{ml})$ was added. After being stirred at -50 to $-60^{\circ} \mathrm{C}$ for $15 \mathrm{~min}$, the mixture was treated with triethylamine $(3.24 \mathrm{ml}, 23.2 \mathrm{mmol})$, stirred at $-60^{\circ} \mathrm{C}$ for $10 \mathrm{~min}$, and then allowed to warm to room temperature over $1 \mathrm{~h}$. The reaction mixture was diluted with ether, washed consecutively with $5 \%$ aqueous $\mathrm{H}_{3} \mathrm{PO}_{4}$, water, and aqueous saturated $\mathrm{NaHCO}_{3}$, dried $\left(\mathrm{MgSO}_{4}\right)$, filtered, and concentrated in vacuo. The residue was used for the next reaction without further purification. $\mathrm{NaH}(242 \mathrm{mg}, 6.05 \mathrm{mmol}, 60 \%$ wt. dispersion in mineral oil) was added to a solution of triethyl phosphonoacetate $(1.26 \mathrm{ml}, 6.35 \mathrm{mmol})$ in THF ( $8 \mathrm{ml})$ under cooling with a water bath. A solution of the residue in THF ( $8 \mathrm{ml})$ was added to the mixture. After being stirred at room temperature for $20 \mathrm{~min}$, the mixture was diluted with ether, washed with water, dried $\left(\mathrm{Na}_{2} \mathrm{SO}_{4}\right)$, filtered, and concentrated in vacuo. The resulting residue was chromatographed on silica gel (hexane-AcOEt, $8: 2$ ) to give $14(1.38 \mathrm{~g}, 93 \%$ yield $)$ as a colorless oil. $[\alpha]_{\mathrm{D}}^{27}+16.8^{\circ}\left(c=5.66, \mathrm{CHCl}_{3}\right)$. IR (neat): $2920(\mathrm{C}-\mathrm{H}), 2850,1722(\mathrm{C}=\mathrm{O}), 1566(\mathrm{C}=\mathrm{C}) \mathrm{cm}^{-1} .{ }^{1} \mathrm{H}-\mathrm{NMR}$ $\left(90 \mathrm{MHz}, \mathrm{CDCl}_{3}\right) \delta: 6.83(1 \mathrm{H}, \mathrm{dd}, J=15.6,6.0 \mathrm{~Hz}, 3-\mathrm{H}), 5.97(1 \mathrm{H}, \mathrm{dd}$, $J=15.8,1.1 \mathrm{~Hz}, 2-\mathrm{H}), 5.51(1 \mathrm{H}, \mathrm{d}, J=5.5 \mathrm{~Hz}, 6-\mathrm{H}), 5.39(1 \mathrm{H}, \mathrm{d}, J=5.3 \mathrm{~Hz}$, $7-\mathrm{H}), 4.62\left(2 \mathrm{H}, \mathrm{s}, \mathrm{OCH}_{2} \mathrm{O}\right), 4.20\left(2 \mathrm{H}, \mathrm{q}, J=7.1 \mathrm{~Hz}, \mathrm{OCH}_{2} \mathrm{CH}_{3}\right), 3.37(3 \mathrm{H}$, s, $\left.\mathrm{OCH}_{3}\right), 2.31(2 \mathrm{H}, \mathrm{m}, 5-\mathrm{H}), 1.99(2 \mathrm{H}, \mathrm{m}, 8-\mathrm{H}), 1.26(\mathrm{~m}), 0.86(6 \mathrm{H}, \mathrm{d}$ $J=6.1 \mathrm{~Hz}, 18-\mathrm{H}$ and $\left.\mathrm{CHCH}_{3}\right)$. FD-MS $m / z(\%): 382\left(\mathrm{M}^{+}, 30\right), 173(100)$ Anal. Calcd for $\mathrm{C}_{23} \mathrm{H}_{42} \mathrm{O}_{4}: \overline{\mathrm{C}}, 72.20 ; \mathrm{H}, 11.06$. Found: C, 71.97; $\mathrm{H}, 11.06$.

(2E,4R,6E)-4-(Methoxymethoxy)-17-methyl-2,6-octadecadien-1-ol (15) A DIBAH solution $(12.7 \mathrm{ml}, 12.7 \mathrm{mmol}, 1.0 \mathrm{M}$ in hexane) was added at $-25^{\circ} \mathrm{C}$ to a solution of $14(1.22 \mathrm{~g}, 3.20 \mathrm{mmol})$ in ether $(12.8 \mathrm{mmol})$. The reaction mixture was allowed to warm to room temperature and stirred at ambient temperature for $12 \mathrm{~h}$. After dropwise addition of a $5 \%$ aqueous $\mathrm{H}_{3} \mathrm{PO}_{4}$ solution, the mixture was extracted with ether. The extracts were acidified with aqueous $5 \% \mathrm{H}_{3} \mathrm{PO}_{4}$, washed with brine and aqueous saturated $\mathrm{NaHCO}_{3}$, dried $\left(\mathrm{Na}_{2} \mathrm{SO}_{4}\right)$, filtered, and concentrated in vacuo. The residue was chromatographed on silica gel (BW-300, hexane-AcOEt, $7: 3)$ to give $15(1.01 \mathrm{~g}, 93 \%$ yield $)$ as a colorless oil. $[\alpha]_{\mathrm{D}}^{27}+30.5^{\circ}(c=0.78$, $\mathrm{CHCl}_{3}$ ). IR (neat): $3420(\mathrm{O}-\mathrm{H}), 2930(\mathrm{C}-\mathrm{H}), 2855 \mathrm{~cm}^{-1} .{ }^{1} \mathrm{H}-\mathrm{NMR}$ $\left(90 \mathrm{MHz}, \mathrm{CDCl}_{3}\right) \delta: 5.71(2 \mathrm{H}, \mathrm{m}, 2-\mathrm{H}$ and $3-\mathrm{H}), 5.46(2 \mathrm{H}, \mathrm{m}, 6-\mathrm{H}$ and $7-\mathrm{H}), 4.69\left(1 \mathrm{H}, \mathrm{d}, J=6.8 \mathrm{~Hz}, \mathrm{OCH}_{\mathrm{a}} \mathrm{O}\right), 4.53\left(1 \mathrm{H}, \mathrm{d}, J=6.6 \mathrm{~Hz}, \mathrm{OCH}_{\mathrm{b}} \mathrm{O}\right)$, $3.35\left(3 \mathrm{H}, \mathrm{s}, \mathrm{OCH}_{3}\right), 2.26(2 \mathrm{H}, \mathrm{m}, 5-\mathrm{H}), 1.99(2 \mathrm{H}, \mathrm{m}, 8-\mathrm{H}), 1.25(\mathrm{~m}), 0.85$ $\left(6 \mathrm{H}, \mathrm{d}, J=6.1 \mathrm{~Hz}, 18-\mathrm{H}\right.$ and $\left.\mathrm{CHCH}_{3}\right)$. FD-MS $m / z(\%): 341\left(\mathrm{M}^{+}+\mathrm{H}\right.$, 2), 131 (100). Anal. Calcd for $\mathrm{C}_{21} \mathrm{H}_{40} \mathrm{O}_{3}: \mathrm{C}, 74.07 ; \mathrm{H}, 11.84$. Found: C, 73.89; H, 11.87 .

$(2 R, 3 R, 4 R, 6 E)-2,3-E p o x y-4$-(methoxymethoxy)-17-methyl-6-octadecen1-ol (6) and Its $(2 S, 3 S, 4 R, 6 E)$ Isomer (16) A mixture of titanium tetraisopropoxide $(325 \mu \mathrm{l}, 1.09 \mathrm{mmol})$ and powdered $4-\AA$ molecular sieves $(400 \mathrm{mg})$ in $\mathrm{CH}_{2} \mathrm{Cl}_{2}$ was cooled to $-30^{\circ} \mathrm{C}$. (-)-Diisopropyl tartrate $(290 \mu \mathrm{l}, 1.37 \mathrm{mmol})$ and a tert-butyl hydroperoxide solution $(1.68 \mathrm{ml}$, $4.10 \mathrm{mmol}, 2.44 \mathrm{M}$ in $\mathrm{CH}_{2} \mathrm{Cl}_{2}$ ) were added dropwise at $-30^{\circ} \mathrm{C}$, sequentially. The reaction mixture was stirred at $-25^{\circ} \mathrm{C}$ for $30 \mathrm{~min}$ and a solution of $15(931 \mathrm{mg}, 2.74 \mathrm{mmol})$ in $\mathrm{CH}_{2} \mathrm{Cl}_{2}(11 \mathrm{ml})$ was added dropwise. The resulting mixture was stored at $-20^{\circ} \mathrm{C}$ for $15 \mathrm{~h}$ in a refrigerator and quenched with water $(6.5 \mathrm{ml})$. The mixture was allowed to warm to room temperature over $1 \mathrm{~h}$ while being stirred vigorously, and filtered through a pad of Celite 545. The filtrate was treated with an aqueous $1 \mathrm{~N} \mathrm{KOH}$ solution $(5.5 \mathrm{ml})$ saturated with $\mathrm{NaCl}$, stirred vigorously for $30 \mathrm{~min}$, and filtered through a pad of Celite 545. The filtrate was extracted with $\mathrm{CHCl}_{3}$. The extracts were washed with water, dried $\left(\mathrm{Na}_{2} \mathrm{SO}_{4}\right)$, filtered, and concentrated in vacuo. The residue was chromatographed on silica gel (hexane-AcOEt, 7:3) to give a mixture of 6 and $16(854 \mathrm{mg}, 88 \%$ yield) as a colorless oil. $[\alpha]_{\mathrm{D}}^{27}+4.65^{\circ}\left(c=2.69, \mathrm{CHCl}_{3}\right)$. IR (neat): $3440(\mathrm{O}-\mathrm{H})$, $2930(\mathrm{C}-\mathrm{H}), 2855 \mathrm{~cm}^{-1}$. FD-MS $m / z(\%): 357\left(\mathrm{M}^{+}+\mathrm{H}, 66\right), 147$ (100). Anal. Calcd for $\mathrm{C}_{21} \mathrm{H}_{40} \mathrm{O}_{4}: \mathrm{C}, 70.74 ; \mathrm{H}, 11.31$. Found: C, 70.56; H, 11.41 .

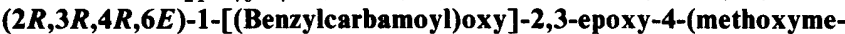
thoxy)-17-methyl-6-octadecene (17) and Its $(2 S, 3 S, 4 R, 6 E)$ Isomer (18) Triethylamine $(962 \mu \mathrm{l}, 6.90 \mathrm{mmol})$ and benzyl isocyanate $(426 \mu \mathrm{l}, 3.45 \mathrm{mmol})$ were added sequentially to a solution of a mixture of 6 and $16(820 \mathrm{mg}$, $2.30 \mathrm{mmol})$ in $\mathrm{CH}_{2} \mathrm{Cl}_{2}(9.2 \mathrm{ml})$. The whole was stirred at room temperature for $15 \mathrm{~h}$, then treated with water $(83 \mu \mathrm{l}, 4.6 \mathrm{mmol})$ and concentrated in vacuo. The residue was chromatographed on silica gel (hexane-AcOEt, $3: 1)$ to give a mixture of 17 and $18(1.09 \mathrm{~g}, 97 \%$ yield $)$ as a colorless oil. $[\alpha]_{\mathrm{D}}^{27}+13.0^{\circ}\left(c=2.66, \mathrm{CHCl}_{3}\right)$. IR $\left(\mathrm{CCl}_{4}\right): 2930(\mathrm{C}-\mathrm{H}), 2855,1735$ $(\mathrm{C}=\mathrm{O}) \mathrm{cm}^{-1}$. FAB-MS m/z (\%): $490\left(\mathrm{M}^{+}+\mathrm{H}, 25\right), 91\left(\mathrm{PhCH}_{2}{ }^{+}, 100\right)$. Anal. Calcd for $\mathrm{C}_{29} \mathrm{H}_{47} \mathrm{NO}_{5}$ : C, 71.13; H, 9.67; N, 2.86. Found: C, 71.39; $\mathrm{H}, 9.69 ; \mathrm{N}, 2.83$.

$\left(4 S, 1^{\prime} S, 2^{\prime} R, 4^{\prime} E\right)-3-B e n z y l-4-\left[1^{\prime}\right.$-hydroxy-2' -(methoxymethoxy)-15'methyl-4'-hexadecenyl]-2-oxazolidinone (19) and Its $\left(4 R, 1^{\prime} R, 2^{\prime} R, 4^{\prime} E\right)$ Isomer (20) A solution of a mixture of 17 and $18(1.03 \mathrm{~g}, 2.11 \mathrm{mmol})$ in THF (42 ml) was added to $\mathrm{NaH}$ (146 mg, $3.65 \mathrm{mmol}, 60 \%$ wt. oil suspension), and the mixture was stirred at $50^{\circ} \mathrm{C}$ for $1 \mathrm{~h}$. Then the reaction was quenched with $5 \%$ aqueous $\mathrm{H}_{3} \mathrm{PO}_{4}$ and the mixture was extracted with $\mathrm{CHCl}_{3}$. The extracts were washed with aqueous saturated $\mathrm{NaHCO}_{3}$, dried $\left(\mathrm{Na}_{2} \mathrm{SO}_{4}\right)$, filtered, and concentrated in vacuo. The residue was chromatographed on silica gel (hexane-AcOEt, $63: 35-6: 4$ ) to give 19 (683 $\mathrm{mg}, 66 \%$ yield) and 20 ( $249 \mathrm{mg}, 24 \%$ yield $)$.

19: Viscous colorless oil. $[\alpha]_{\mathrm{D}}^{27}-19.3^{\circ}\left(c=2.02, \mathrm{CHCl}_{3}\right)$. IR $\left(\mathrm{CCl}_{4}\right)$ : $3380(\mathrm{O}-\mathrm{H}), 2930(\mathrm{C}-\mathrm{H}), 2855,1738(\mathrm{C}=\mathrm{O}) \mathrm{cm}^{-1} .{ }^{1} \mathrm{H}-\mathrm{NMR}(270 \mathrm{MHz}$, $\left.\mathrm{CDCl}_{3}\right) \delta: 7.32(5 \mathrm{H}, \mathrm{m}$, aromatic $\mathrm{H}), 5.40\left(1 \mathrm{H}, \mathrm{dt}, J=14.6,7.1 \mathrm{~Hz}, 4^{\prime}-\mathrm{H}\right)$, $5.22\left(1 \mathrm{H}, \mathrm{dt}, J=14.8,7.3 \mathrm{~Hz}, 5^{\prime}-\mathrm{H}\right), 4.70\left(1 \mathrm{H}, \mathrm{d}, J=15.5 \mathrm{~Hz}\right.$, benzylic $\left.\mathrm{H}_{\mathrm{a}}\right)$, $4.57\left(1 \mathrm{H}, \mathrm{d}, J=6.4 \mathrm{~Hz}, \mathrm{OCH}_{\mathrm{a}} \mathrm{O}\right), 4.53\left(1 \mathrm{H}, \mathrm{d}, J=6.6 \mathrm{~Hz}, \mathrm{OCH}_{\mathrm{b}} \mathrm{O}\right), 4.47$ $\left(1 \mathrm{H}, \mathrm{dd}, J=8.9,7.4 \mathrm{~Hz}, 5-\mathrm{H}_{\mathrm{a}}\right), 4.25\left(1 \mathrm{H}, \mathrm{d}, J=15.2 \mathrm{~Hz}\right.$, benzylic $\left.\mathrm{H}_{\mathrm{b}}\right), 4.23$ $\left(1 \mathrm{H}, \mathrm{t}, J=8.9 \mathrm{~Hz}, 5-\mathrm{H}_{\mathrm{b}}\right), 3.90(1 \mathrm{H}, \mathrm{dd}, J=7.5,1.5 \mathrm{~Hz}, 4-\mathrm{H}), 3.81(1 \mathrm{H}, \mathrm{m}$, $\left.1^{\prime}-\mathrm{H}\right), 3.57\left(\mathrm{lH}, \mathrm{dd}, J=5.8,5.8 \mathrm{~Hz}, 2^{\prime}-\mathrm{H}\right), 3.29\left(3 \mathrm{H}, \mathrm{s}, \mathrm{OCH}_{3}\right), 2.87(1 \mathrm{H}$, d, $J=5.3 \mathrm{~Hz}, \mathrm{OH}), 2.12\left(2 \mathrm{H}, \mathrm{m}, 3^{\prime}-\mathrm{H}\right), 1.95\left(2 \mathrm{H}, \mathrm{m}, 6^{\prime}-\mathrm{H}\right), 1.51(1 \mathrm{H}, \mathrm{dqq}$, $\left.J=6.5,6.5,6.5 \mathrm{~Hz}, 15^{\prime}-\mathrm{H}\right), 1.26(\mathrm{~m}), 0.86\left(6 \mathrm{H}, \mathrm{d}, J=6.6 \mathrm{~Hz}, 16^{\prime}-\mathrm{H}\right.$ and $\left.\mathrm{CHCH}_{3}\right)$. FD-MS $m / z(\%): 490\left(\mathrm{M}^{+}+\mathrm{H}, 98\right), 489\left(\mathrm{M}^{+}, 100\right)$. Anal. Calcd for $\mathrm{C}_{29} \mathrm{H}_{47} \mathrm{NO}_{5}$ : C, 71.13; H, 9.67; N, 2.86. Found: C, 71.01; H, 9.75; N, 2.71.

20: Viscous colorless oil. $[\alpha]_{\mathrm{D}}^{27}+5.99^{\circ}(c=1.74, \mathrm{CHCl})_{3}$. IR $\left(\mathrm{CCl}_{4}\right)$ : $3410(\mathrm{O}-\mathrm{H}), 2930(\mathrm{C}-\mathrm{H}), 2855,1740(\mathrm{C}=\mathrm{O}) \mathrm{cm}^{-1} .{ }^{1} \mathrm{H}-\mathrm{NMR}(270 \mathrm{MHz}$, $\left.\mathrm{CDCl}_{3}\right) \delta: 7.32(5 \mathrm{H}, \mathrm{m}$, aromatic $\mathrm{H}), 5.40\left(1 \mathrm{H}, \mathrm{dt}, J=14.6,7.1 \mathrm{~Hz}, 4^{\prime}-\mathrm{H}\right)$, $5.20\left(1 \mathrm{H}, \mathrm{dt}, J=14.7,7.4 \mathrm{~Hz}, 5^{\prime}-\mathrm{H}\right), 4.80\left(1 \mathrm{H}, \mathrm{d}, J=15.2 \mathrm{~Hz}\right.$, benzylic $\left.\mathrm{H}_{\mathrm{a}}\right)$, $4.64\left(1 \mathrm{H}, \mathrm{d}, J=6.9 \mathrm{~Hz}, \mathrm{OCH}_{\mathrm{a}} \mathrm{O}\right), 4.60\left(1 \mathrm{H}, \mathrm{d}, J=6.8 \mathrm{~Hz}, \mathrm{OCH}_{\mathrm{b}} \mathrm{O}\right), 4.48$ $\left(1 \mathrm{H}, \mathrm{dd}, J=8.7,6.8 \mathrm{~Hz}, 5-\mathrm{H}_{\mathrm{a}}\right), 4.21\left(1 \mathrm{H}, \mathrm{t}, J=8.8 \mathrm{~Hz}, 5-\mathrm{H}_{\mathrm{b}}\right), 4.18(1 \mathrm{H}, \mathrm{d}$, $J=15.7 \mathrm{~Hz}$, benzylic $\left.\mathrm{H}_{\mathrm{b}}\right), 3.87\left(1 \mathrm{H}, \mathrm{m}, 1^{\prime}-\mathrm{H}\right), 3.71(1 \mathrm{H}, \mathrm{dd}, J=7.1,1.7 \mathrm{~Hz}$, $4-\mathrm{H}), 3.34\left(3 \mathrm{H}, \mathrm{s}, \mathrm{OCH}_{3}\right), 3.15(1 \mathrm{H}, \mathrm{d}, J=3.6 \mathrm{~Hz}, \mathrm{OH}), 2.25\left(2 \mathrm{H}, \mathrm{m}, 3^{\prime}-\mathrm{H}\right)$, $2.11\left(2 \mathrm{H}, \mathrm{m}, 6^{\prime}-\mathrm{H}\right), 1.51\left(1 \mathrm{H}, \mathrm{dqq}, J=6.6,6.6,6.6 \mathrm{~Hz}, 15^{\prime}-\mathrm{H}\right), 1.26(\mathrm{~m})$, $0.86\left(6 \mathrm{H}, \mathrm{d}, J=6.6 \mathrm{~Hz}, 16^{\prime}-\mathrm{H}\right.$ and $\left.\mathrm{CHCH}_{3}\right)$. FD-MS $m / z(\%): 490$ $\left(\mathrm{M}^{+}+\mathrm{H}, 93\right), 489\left(\mathrm{M}^{+}, 100\right)$. Anal. Calcd for $\mathrm{C}_{29} \mathrm{H}_{47} \mathrm{NO}_{5}: \mathrm{C}, 71.13 ; \mathrm{H}$, 9.67; N, 2.86. Found: C, 71.01; H, 9.61; N, 2.84 .

$\left(4 S, 1^{\prime} S, 2^{\prime} R, 4^{\prime} E\right)-4-\left[1^{\prime}-H y d r o x y-2 '\right.$-(methoxymethoxy)-15'-methyl-4' hexadecenyl]-2-oxazolidinone (21) Liquid EtNH $\mathrm{EH}_{2}(30 \mathrm{ml})$ was added at $0{ }^{\circ} \mathrm{C}$ to a solution of $19(631 \mathrm{mg}, 5.09 \mathrm{mmol})$ in tert-BuOH $(6.3 \mathrm{ml})$. Several pieces of lithium $(100 \mathrm{mg})$ were added at $-78^{\circ} \mathrm{C}$ to the mixture. The blue-colored mixture was stirred at $-78^{\circ} \mathrm{C}$ for $10 \mathrm{~min}$ and the reaction 
was quenched with ammonium chloride. After removal of the unreacted lithium, the mixture was concentrated to half of the original volume, diluted with ether, washed with brine, dried $\left(\mathrm{Na}_{2} \mathrm{SO}_{4}\right)$, filtered, and concentrated in vacuo. The residue was chromatographed on silica gel (hexane-AcOEt, $3: 7$ ) to give $21(356 \mathrm{mg}, 69 \%$ yield) as a colorless oil. $[\alpha]_{\mathrm{D}}^{27}-26.6^{\circ}\left(c=2.08, \mathrm{CHCl}_{3}\right)$. IR $\left(\mathrm{CCl}_{4}\right): 3400$ and $3280(\mathrm{O}-\mathrm{H}$ and $\mathrm{N}-\mathrm{H}), 2930(\mathrm{C}-\mathrm{H}), 2850,1752(\mathrm{C}=\mathrm{O}) \mathrm{cm}^{-1} \cdot{ }^{1} \mathrm{H}-\mathrm{NMR}(270 \mathrm{MHz}$, $\left.\mathrm{CDCl}_{3}\right) \delta: 6.37(1 \mathrm{H}, \mathrm{s}, \mathrm{NH}), 5.54\left(1 \mathrm{H}, \mathrm{dt}, J=14.4,7.0 \mathrm{~Hz}, 4^{\prime}-\mathrm{H}\right), 5.39$ $\left(1 \mathrm{H}, \mathrm{dt}, J=14.7,7.2 \mathrm{~Hz}, 5^{\prime}-\mathrm{H}\right), 4.63\left(2 \mathrm{H}, \mathrm{s}, \mathrm{OCH}_{2} \mathrm{O}\right), 4.52(1 \mathrm{H}, \mathrm{dd}, J=8.9$, $\left.6.3 \mathrm{~Hz}, 5 \mathrm{H}_{\mathrm{a}}\right), 4.41\left(1 \mathrm{H}, \mathrm{t}, J=8.7 \mathrm{~Hz}, 5-\mathrm{H}_{\mathrm{b}}\right), 4.08(1 \mathrm{H}, \mathrm{m}, 4-\mathrm{H}), 3.68(1 \mathrm{H}, \mathrm{t}$, $\left.J=4.6 \mathrm{~Hz}, 1^{\prime}-\mathrm{H}\right), 3.61\left(1 \mathrm{H}, \mathrm{dt}, J=3.1,3.1 \mathrm{~Hz}, 2^{\prime}-\mathrm{H}\right), 3.37\left(3 \mathrm{H}, \mathrm{s}, \mathrm{OCH}_{3}\right)$, $2.31\left(2 \mathrm{H}, \mathrm{m}, 3^{\prime}-\mathrm{H}\right), 2.00\left(2 \mathrm{H}, \mathrm{m}, 6^{\prime}-\mathrm{H}\right), 1.51(1 \mathrm{H}, \mathrm{dqq}, J=6.6,6.6,6.6 \mathrm{~Hz}$, $\left.15^{\prime}-\mathrm{H}\right), 1.26(\mathrm{~m}), 0.86\left(6 \mathrm{H}, \mathrm{d}, J=6.6 \mathrm{~Hz}, 16^{\prime}-\mathrm{H}\right.$ and $\left.\mathrm{CHCH}_{3}\right)$. FD-MS $m / z$ (\%): $400\left(\mathrm{M}^{+}+\mathrm{H}, 100\right)$. Anal. Calcd for $\mathrm{C}_{22} \mathrm{H}_{41} \mathrm{NO}_{5}$ : C, 66.13; H, 10.34; $\mathrm{N}, 3.51$. Found: C, 65.91; H, 10.43; N, 3.50.

$(2 S, 3 S, 4 R, 6 E)-2$-Amino-17-methyl-6-octadecene-1,3,4-triol (5) An aqueous $2 \mathrm{~N} \mathrm{KOH}$ solution $(3.52 \mathrm{ml}, 7.04 \mathrm{mmol})$ was added to a solution of $21(282 \mathrm{mg}, 70.6 \mu \mathrm{mol})$ in EtOH $(3.5 \mathrm{ml})$. The reaction mixture was heated for $5 \mathrm{~h}$ at reflux, cooled to room temperature, and extracted with $\mathrm{Et}_{2} \mathrm{O}$. The extracts were dried over $\mathrm{Na}_{2} \mathrm{SO}_{4}$, filtered, and concentrated in vacuo. The residue $(268 \mathrm{mg})$ was dissolved in $2 \mathrm{~N} \mathrm{HCl}$ in $\mathrm{MeOH}(2 \mathrm{ml})$. The resulting mixture was heated for $5 \mathrm{~h}$ at reflux and alkalinized with a $0.1 \mathrm{M}$ solution of $\mathrm{K}_{2} \mathrm{CO}_{3}$ in $\mathrm{MeOH}$. The precipitate was filtered off through a cotton plug. The filtrate was concentrated by blowing a stream of nitrogen gas over it. The residue was chromatographed on silica gel (BW-300, $\left.\mathrm{CHCl}_{3}-\mathrm{MeOH}-\mathrm{H}_{2} \mathrm{O}, 7: 3: 0.5\right)$ to give crude $5(515 \mathrm{mg})$, which was passed through a Sephadex LH-20 column (MeOH as the eluent). The obtained $5\left(212 \mathrm{mg}, 91 \%\right.$ yield) was a viscous colorless oil. ${ }^{1} \mathrm{H}-\mathrm{NMR}(270 \mathrm{MHz}$, $\left.\mathrm{C}_{5} \mathrm{D}_{5} \mathrm{~N}\right) \delta: 5.91(1 \mathrm{H}, \mathrm{dt}, J=14.8,7.3 \mathrm{~Hz}, 6-\mathrm{H}), 5.67(1 \mathrm{H}, \mathrm{dt}, J=14.6$, $7.1 \mathrm{~Hz}, 7-\mathrm{H}), 4.78(1 \mathrm{H}$, dd, $J=8.7,3.3 \mathrm{~Hz}, 3-\mathrm{H}), 4.72(2 \mathrm{H}, \mathrm{m}, 1-\mathrm{H}), 4.27$ $(1 \mathrm{H}, \mathrm{dt}, J=8.4,2.8 \mathrm{~Hz}, 4-\mathrm{H}), 3.05\left(1 \mathrm{H}, \mathrm{m}, 5-\mathrm{H}_{\mathrm{a}}\right), 2.61$ (1H, quint., $\left.J=7.3 \mathrm{~Hz}, 5-\mathrm{H}_{\mathrm{b}}\right), 2.00(2 \mathrm{H}, \mathrm{q}, J=6.6 \mathrm{~Hz}, 8-\mathrm{H}), 1.24(\mathrm{~m}), 0.87(6 \mathrm{H}, \mathrm{d}$, $J=6.6 \mathrm{~Hz}, 18-\mathrm{H}$ and $\left.\mathrm{CHCH}_{3}\right) .{ }^{13} \mathrm{C}-\mathrm{NMR}\left(67.8 \mathrm{MHz}, \mathrm{C}_{5} \mathrm{D}_{5} \mathrm{~N}\right) \delta: 133.2$ (d, (C7)), $127.4(\mathrm{~d},(\mathrm{C} 6)), 73.4(\mathrm{~d},(\mathrm{C} 3$ and C4)), $60.0(\mathrm{t},(\mathrm{C} 1)), 56.7$ (d, (C2)), $39.2(\mathrm{t}), 38.6(\mathrm{t},(\mathrm{C} 5)), 33.1(\mathrm{t},(\mathrm{C} 8)), 30.2(\mathrm{t}), 30.0(\mathrm{t}), 29.9(\mathrm{t}), 29.8$ $(\mathrm{t}), 29.6(\mathrm{t}), 28.2(\mathrm{~d},(\mathrm{Cl})), 27.7(\mathrm{t}), 22.8\left(\mathrm{q},\left(\mathrm{C} 18\right.\right.$ and $\left.\left.\mathrm{CHCH}_{3}\right)\right)$. FD-MS $\mathrm{m} / \mathrm{z}(\%): 330\left(\mathrm{M}^{+}+\mathrm{H}, 100\right)$. This product was used in the next reaction without further purification.

(2S,3S,4R,6E)-2-Acetamido-17-methyl-1,3,4-triacetoxy-6-octadecene (23) Acetic anhydride $(147 \mu \mathrm{l}, 1.56 \mathrm{mmol})$ and pyridine $(127 \mu \mathrm{l}, 1.57$ mmol) were added to $5(34.3 \mathrm{mg}, 104 \mu \mathrm{mol})$. The reaction mixture was stirred at $50^{\circ} \mathrm{C}$ for $1 \mathrm{~h}$ and at room temperature for $15 \mathrm{~h}$, then concentrated by blowing nitrogen gas over it. The residue was chromatographed on silica gel (BW-300, hexane-AcOEt, $2: 3)$ to give $23(26.8 \mathrm{mg}, 52 \%$ yield) as a colorless viscous oil. $[\alpha]_{\mathrm{D}}^{26}+9.76^{\circ}(c=1.24, \mathrm{CHCl}) . \mathrm{IR}\left(\mathrm{CCl}_{4}\right): 3370$ $(\mathrm{N}-\mathrm{H}), 2930(\mathrm{C}-\mathrm{H}), 2855,1755(\mathrm{OC}=\mathrm{O}), 1690(\mathrm{NC}=\mathrm{O}) \mathrm{cm}^{-1} \cdot{ }^{1} \mathrm{H}-\mathrm{NMR}$ $\left(270 \mathrm{MHz}, \mathrm{CDCl}_{3}\right) \delta: 6.06(1 \mathrm{H}, \mathrm{d}, J=9.4 \mathrm{~Hz}, \mathrm{NH}), 5.49(1 \mathrm{H}, \mathrm{dt}, J=14.7$, $7.2 \mathrm{~Hz}, 6-\mathrm{H}), 5.28(1 \mathrm{H}, \mathrm{dt}, J=14.7,7.3 \mathrm{~Hz}, 7-\mathrm{H}), 5.09(1 \mathrm{H}, \mathrm{dd}, J=7.6$, $3.4 \mathrm{~Hz}, 3-\mathrm{H}), 4.96(1 \mathrm{H}, \mathrm{dt}, J=9.1,3.8 \mathrm{~Hz}, 4-\mathrm{H}), 4.51(1 \mathrm{H}, \mathrm{m}, 2-\mathrm{H}), 4.26$ $\left(1 \mathrm{H}, \mathrm{dd}, J=11.7,5.0 \mathrm{~Hz}, 1-\mathrm{H}_{\mathrm{a}}\right), 4.01\left(1 \mathrm{H}, \mathrm{dd}, J=11.6,3.4 \mathrm{~Hz}, 1-\mathrm{H}_{\mathrm{b}}\right), 2.43$ $\left(1 \mathrm{H}, \mathrm{m}, 5-\mathrm{H}_{\mathrm{a}}\right), 2.28\left(1 \mathrm{H}, \mathrm{m}, 5-\mathrm{H}_{\mathrm{b}}\right), 2.09\left(3 \mathrm{H}, \mathrm{s}, \mathrm{CH}_{3} \mathrm{CO}\right), 2.05(3 \mathrm{H}, \mathrm{s}$, $\left.\mathrm{CH}_{3} \mathrm{CO}\right), 2.04$ (3H, s, $\left.\mathrm{CH}_{3} \mathrm{CO}\right), 2.02\left(3 \mathrm{H}, \mathrm{s}, \mathrm{CH}_{3} \mathrm{CO}\right), 1.96(2 \mathrm{H}, \mathrm{m}, 8-\mathrm{H})$, $1.51(1 \mathrm{H}, \mathrm{dqq}, J=6.6,6.6,6.6 \mathrm{~Hz}, 17-\mathrm{H}), 1.25(\mathrm{~m}), 0.86(6 \mathrm{H}, \mathrm{d}, J=6.6 \mathrm{~Hz}$,

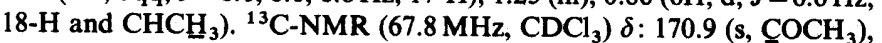
$170.2\left(\mathrm{~s}, \mathrm{COCH}_{3}\right), 169.7\left(\mathrm{~s}, \mathrm{COCH}_{3}\right), 134.7$ (d, (C6)), 124.0 (d, (C7)), 72.5 $(\mathrm{d},(\mathrm{C} 4)), 72.2(\mathrm{~d},(\mathrm{C} 3)), 62.8(\mathrm{t},(\mathrm{C} 1)), 47.7(\mathrm{~d},(\mathrm{C} 2)), 39.1(\mathrm{t}), 32.6(\mathrm{t})$, $32.1(\mathrm{t},(\mathrm{C} 5)), 30.0(\mathrm{t}), 29.7(\mathrm{t}), 29.6(\mathrm{t}), 29.5(\mathrm{t}), 29.4(\mathrm{t}), 29.2(\mathrm{t}), 28.0(\mathrm{~d}$, $(\mathrm{C} 17)), 27.4(\mathrm{t}), 23.3\left(\mathrm{q}, \mathrm{COCH}_{3}\right), 22.7\left(\mathrm{q},\left(\mathrm{Cl}\right.\right.$ and $\left.\left.\mathrm{CHCH}_{3}\right)\right), 21.0(\mathrm{q}$, $\left.\mathrm{COCH}_{3}\right), 20.8\left(\mathrm{q}, \mathrm{COCH}_{3}\right)$. FD-MS m/z (\%): $498\left(\mathrm{M}^{+}+\mathrm{H}, 100\right)$. Anal. Calcd for $\mathrm{C}_{27} \mathrm{H}_{47} \mathrm{NO}_{7}: \mathrm{C}, 65.16 ; \mathrm{H}, 9.52 ; \mathrm{N}, 2.81$. Found: $\mathrm{C}, 64.83 ; \mathrm{H}$, $9.40 ; \mathrm{N}, 2.77$

(2S,3S,4R)-2-Acetamido-17-methyl-1,3,4-triacetoxyoctadecane (25) A suspension of $23(23.8 \mathrm{mg}, 47.8 \mu \mathrm{mol})$ and $10 \%$ palladium on carbon $(22.4 \mathrm{mg})$ in $\mathrm{MeOH}(2.4 \mathrm{ml})$ was stirred for $7 \mathrm{~h}$ under a hydrogen atmosphere. The mixture was filtered through a pad of Celite 545 and concentrated in vacuo. The residue was chromatographed on silica gel (BW-300, hexane-AcOEt, 2:3) to give $25(14.2 \mathrm{mg}, 59 \%$ yield) as a colorless oil. $[\alpha]_{\mathrm{D}}^{26}+26.1^{\circ}\left(c=0.71, \mathrm{CHCl}_{3}\right)$. IR $\left(\mathrm{CCl}_{4}\right): 3350(\mathrm{~N}-\mathrm{H}), 2930$ $(\mathrm{C}-\mathrm{H}), 2855,1750(\mathrm{OC}=\mathrm{O}), 1690(\mathrm{NC}=\mathrm{O}) \mathrm{cm}^{-1} .{ }^{1} \mathrm{H}-\mathrm{NMR}(270 \mathrm{MHz}$, $\left.\mathrm{CDCl}_{3}\right) \delta: 6.00(1 \mathrm{H}, \mathrm{d}, J=9.4 \mathrm{~Hz}, \mathrm{NH}), 5.11(1 \mathrm{H}, \mathrm{dd}, J=8.3,3.1 \mathrm{~Hz}$ 3-H), 4.93 (1H, dt, $J=9.3,3.5 \mathrm{~Hz}, 4-\mathrm{H}), 4.47(1 \mathrm{H}, \mathrm{m}, 2-\mathrm{H}), 4.29$ (1H, dd, $\left.J=11.6,4.9 \mathrm{~Hz}, 1-\mathrm{H}_{\mathrm{a}}\right), 4.00\left(1 \mathrm{H}, \mathrm{dd}, J=11.6,3.1 \mathrm{~Hz}, 1-\mathrm{H}_{\mathrm{b}}\right), 2.08(3 \mathrm{H}, \mathrm{s}$, $\left.\mathrm{CH}_{3} \mathrm{CO}\right), 2.05\left(6 \mathrm{H}, \mathrm{s}, \mathrm{CH}_{3} \mathrm{CO} \times 2\right), 2.03\left(3 \mathrm{H}, \mathrm{s}, \mathrm{CH}_{3} \mathrm{CO}\right), 1.51$ (1H, dqq, $J=6.6,6.6,6.6 \mathrm{~Hz}, 17-\mathrm{H}), 1.25(\mathrm{~m}), 0.86(6 \mathrm{H}, \mathrm{d}, J=6.6 \mathrm{~Hz}, 18-\mathrm{H}$ and $\left.\mathrm{CHCH}_{3}\right) .{ }^{13} \mathrm{C}-\mathrm{NMR}\left(67.8 \mathrm{MHz}, \mathrm{CDCl}_{3}\right) \delta: 171.2\left(\mathrm{~s}, \mathrm{COCH}_{3}\right), 170.9(\mathrm{~s}$,
$\mathrm{COCH}_{3}$ ), $170.1\left(\mathrm{~s}, \mathrm{COCH}_{3}\right), 169.7\left(\mathrm{~s}, \mathrm{COCH}_{3}\right), 73.0$ (d, (C3)), 72.0 (d, (C4)), $62.9(\mathrm{t},(\mathrm{Cl})), 47.6(\mathrm{~d},(\mathrm{C} 2)), 39.1(\mathrm{t}), 30.0(\mathrm{t}), 29.7(\mathrm{t}), 29.6(\mathrm{t}), 29.5$ (t), 29.3 (t), 28.2 (d), 28.0 (d, (C17)), $27.4(\mathrm{t}), 25.5$ (d), $23.3\left(\mathrm{q}, \mathrm{COCH}_{3}\right.$ ), 22.7 (q, (C18 and $\left.\left.\mathrm{CHCH}_{3}\right)\right), 21.0\left(\mathrm{q}, \mathrm{COCH}_{3}\right), 20.7\left(\mathrm{q}, \mathrm{COCH}_{3}\right)$. FD-MS $m / z(\%): 500\left(\mathrm{M}^{+}+\mathrm{H}, 100\right)$. Anal. Calcd for $\mathrm{C}_{27} \mathrm{H}_{49} \mathrm{NO}_{7}: \mathrm{C}, 64.90 ; \mathrm{H}$, 9.88; N, 2.80. Found: C, 64.51; H, 9.77; N, 2.71 .

$(2 R, 3 R, 4 R, 6 E)-2$-Amino-17-methyl-6-octadecene-1,3,4-triol (22) According to the procedures described for the preparation of 5 from 19, compound 20 (332 mg) was converted into $22(120 \mathrm{mg}, 54 \%$ yield) as a colorless viscous oil. ${ }^{1} \mathrm{H}-\mathrm{NMR}\left(270 \mathrm{MHz}, \mathrm{C}_{5} \mathrm{D}_{5} \mathrm{~N}\right) \delta: 5.64(1 \mathrm{H}$, dt, $J=14.5,7.1 \mathrm{~Hz}, 7-\mathrm{H}), 2.78(2 \mathrm{H}, \mathrm{t}, J=6.7 \mathrm{~Hz}, 5-\mathrm{H}), 2.00(2 \mathrm{H}, \mathrm{q}, J=6.8 \mathrm{~Hz}$, $8-\mathrm{H}), 1.49(\mathrm{lH}, \mathrm{dqq}, J=6.6,6.6,6.6 \mathrm{~Hz}, 17-\mathrm{H}), 1.25(\mathrm{~m}), 0.87(6 \mathrm{H}, \mathrm{d}$, $J=6.6 \mathrm{~Hz}, 18-\mathrm{H}$ and $\left.\mathrm{CHCH}_{3}\right)$. FD-MS $m / z(\%): 330\left(\mathrm{M}^{+}+\mathrm{H}, 100\right)$. This product was used in the next reaction without further purification.

$(2 R, 3 R, 4 R, 6 E)-2$-Acetamido-17-methyl-1,3,4-triacetoxy-6-octadecene (24) According to the procedures described for the preparation of 23 , compound $22(49.1 \mathrm{mg})$ was converted into $24(70.1 \mathrm{mg}, 95 \%$ yield) as a colorless oil. $[\alpha]_{\mathrm{D}}^{27}-2.14^{\circ}\left(c=1.00, \mathrm{CHCl}_{3}\right)$. IR $\left(\mathrm{CCl}_{4}\right): 3320(\mathrm{~N}-\mathrm{H}), 2930$ $(\mathrm{C}-\mathrm{H}), 2855,1745(\mathrm{OC}=\mathrm{O}), 1695(\mathrm{NC}=\mathrm{O}) \mathrm{cm}^{-1} \cdot{ }^{1} \mathrm{H}-\mathrm{NMR}(270 \mathrm{MHz}$, $\left.\mathrm{CDCl}_{3}\right) \delta: 5.73(1 \mathrm{H}, \mathrm{d}, J=9.9 \mathrm{~Hz}, \mathrm{NH}), 5.48(1 \mathrm{H}, \mathrm{dt}, J=14.6,7.1 \mathrm{~Hz}$, 6-H), $5.27(1 \mathrm{H}, \mathrm{dt}, J=14.9,7.4 \mathrm{~Hz}, 7-\mathrm{H}), 4.54(1 \mathrm{H}, \mathrm{m}, 2-\mathrm{H}), 4.22(1 \mathrm{H}$, dd, $\left.J=11.7,4.5 \mathrm{~Hz}, 1-\mathrm{H}_{\mathrm{a}}\right), 3.96\left(1 \mathrm{H}, \mathrm{dd}, J=11.7,3.3 \mathrm{~Hz}, 1-\mathrm{H}_{\mathrm{b}}\right), 2.13(3 \mathrm{H}$, s, $\left.\mathrm{CH}_{3} \mathrm{CO}\right), 2.08\left(3 \mathrm{H}, \mathrm{s}, \mathrm{CH}_{3} \mathrm{CO}\right), 2.07\left(3 \mathrm{H}, \mathrm{s}, \mathrm{CH}_{3} \mathrm{CO}\right), 1.98(3 \mathrm{H}, \mathrm{s}$, $\left.\mathrm{CH}_{3} \mathrm{CO}\right), 1.51(1 \mathrm{H}, \mathrm{dqq}, J=6.6,6.6,6.6 \mathrm{~Hz}, 17-\mathrm{H}), 1.25(\mathrm{~m}), 0.86(6 \mathrm{H}$, d, $J=6.6 \mathrm{~Hz}, 18-\mathrm{H}$ and $\left.\mathrm{CHCH}_{3}\right) .{ }^{13} \mathrm{C}-\mathrm{NMR}\left(67.8 \mathrm{MHz}, \mathrm{CDCl}_{3}\right) \delta: 170.8$ $\left(\mathrm{s}, \mathrm{COCH}_{3}\right), 170.4\left(\mathrm{~s}, \mathrm{COCH}_{3}\right), 170.3\left(\mathrm{~s}, \mathrm{COCH}_{3}\right), 169.6\left(\mathrm{~s}, \mathrm{COCH}_{3}\right)$, 135.3 (d, (C6)), 123.3 (d, (C7)), 71.6 (d, (C4)), 70.9 (d, (C3)), 63.1 (t, (C1)), $47.5(\mathrm{~d},(\mathrm{C} 2)), 39.1(\mathrm{t}), 34.4(\mathrm{t},(\mathrm{C} 5)), 32.6(\mathrm{t}), 29.9(\mathrm{t}), 29.7(\mathrm{t}), 29.5(\mathrm{t})$, $29.4(\mathrm{t}), 29.2(\mathrm{t}), 28.0(\mathrm{~d},(\mathrm{Cl})), 27.4\left(\mathrm{q}, \mathrm{COCH} \mathrm{C}_{3}\right), 23.3\left(\mathrm{q}, \mathrm{COCH}_{3}\right), 22.7$ (q, $\left(\mathrm{Cl} 8\right.$ and $\left.\mathrm{CHCH}_{3}\right)$ ), $21.0\left(\mathrm{q}, \mathrm{COCH}_{3}\right), 20.8\left(\mathrm{q}, \mathrm{COCH}_{3}\right)$. FD-MS m/z (\%): $498\left(\mathrm{M}^{+}+\mathrm{H}, 100\right)$. Anal. Calcd for $\mathrm{C}_{27} \mathrm{H}_{47} \mathrm{NO}_{7}: \mathrm{C}, 65.16 ; \mathrm{H}, 9.52$; N, 2.81. Found: C, 64.97; H, 9.43; N, 2.75.

$(2 R, 3 R, 4 R)-2$-Acetamido-17-methyl-1,3,4-triacetoxyoctadecane (26) According to the procedures described for the preparation of 25 , compound $24(36.3 \mathrm{mg})$ was converted into $26(21.7 \mathrm{mg}, 60 \%$ yield) as a colorless powder. mp $77^{\circ} \mathrm{C}$. $[\alpha]_{\mathrm{D}}^{27}-3.09^{\circ}\left(c=1.09, \mathrm{CHCl}_{3}\right)$. IR $\left(\mathrm{CCl}_{4}\right)$ : $3380(\mathrm{~N}-\mathrm{H}), 2920(\mathrm{C}-\mathrm{H}), 2850,1745(\mathrm{OC}=\mathrm{O}), 1690(\mathrm{NC}=\mathrm{O}) \mathrm{cm}^{-1}$. ${ }^{1} \mathrm{H}-\mathrm{NMR}\left(270 \mathrm{MHz} \mathrm{CDCl}_{3}\right) \delta: 5.79(1 \mathrm{H}, \mathrm{d}, J=9.7 \mathrm{~Hz}, \mathrm{NH}), 5.09(2 \mathrm{H}$, $\mathrm{m}, 3-\mathrm{H}$ and $4-\mathrm{H}), 4.53(1 \mathrm{H}, \mathrm{m}, 2-\mathrm{H}), 4.23\left(1 \mathrm{H}, \mathrm{dd}, J=11.7,4.6 \mathrm{~Hz}, 1-\mathrm{H}_{\mathrm{a}}\right)$, $3.97\left(1 \mathrm{H}, \mathrm{dd}, J=11.7,3.3 \mathrm{~Hz}, 1-\mathrm{H}_{\mathrm{b}}\right), 2.13\left(3 \mathrm{H}, \mathrm{s}, \mathrm{CH}_{3} \mathrm{CO}\right), 2.09(6 \mathrm{H}, \mathrm{s}$, $\left.\mathrm{CH}_{3} \mathrm{CO} \times 2\right), 2.07\left(3 \mathrm{H}, \mathrm{s}, \mathrm{CH}_{3} \mathrm{CO}\right), 1.98\left(3 \mathrm{H}, \mathrm{s}, \mathrm{CH}_{3} \mathrm{CO}\right), 1.51(1 \mathrm{H}, \mathrm{dqq}$, $J=6.6,6.6,6.6 \mathrm{~Hz}, 17-\mathrm{H}), 1.24(\mathrm{~m}), 0.86(6 \mathrm{H}, \mathrm{d}, J=6.6 \mathrm{~Hz}, 18-\mathrm{H}$ and $\left.\mathrm{CHCH}_{3}\right)$. ${ }^{1} \mathrm{H}-\mathrm{NMR}\left(400 \mathrm{MHz}, \mathrm{C}_{6} \mathrm{D}_{6}\right) \delta: 5.30(\mathrm{HH}, \mathrm{d}, J=9.9 \mathrm{~Hz}, \mathrm{NH})$, $5.23(1 \mathrm{H}$, ddd, $J=8.1,5.4,2.6 \mathrm{~Hz}, 4-\mathrm{H}), 4.80(1 \mathrm{H}, \mathrm{ddt}, J=9.6,5.1,3.2 \mathrm{~Hz}$, $3-\mathrm{H}), 4.25\left(1 \mathrm{H}, \mathrm{dd}, J=11.7,5.1 \mathrm{~Hz}, 1-\mathrm{H}_{\mathrm{a}}\right), 3.90(1 \mathrm{H}, \mathrm{dd}, J=11.7,3.2 \mathrm{~Hz}$, $\left.1-\mathrm{H}_{\mathrm{b}}\right), 1.99\left(3 \mathrm{H}, \mathrm{s}, \mathrm{CH}_{3} \mathrm{CO}\right), 1.78\left(6 \mathrm{H}, \mathrm{s}, \mathrm{CH}_{3} \mathrm{CO} \times 2\right), 1.73(3 \mathrm{H}, \mathrm{s}$, $\left.\mathrm{CH}_{3} \mathrm{CO}\right), 1.66\left(3 \mathrm{H}, \mathrm{s}, \mathrm{CH}_{3} \mathrm{CO}\right), 1.31(\mathrm{~m}), 0.91(6 \mathrm{H}, \mathrm{d}, J=6.6 \mathrm{~Hz}, 18-\mathrm{H}$ and $\left.\mathrm{CHCH}_{3}\right) .{ }^{13} \mathrm{C}-\mathrm{NMR}\left(67.8 \mathrm{MHz}^{\mathrm{C}} \mathrm{CDCl}_{3}\right) \delta: 170.7\left(\mathrm{~s}, \mathrm{COCH}_{3}\right), 170.6$ $\left(\mathrm{s}, \mathrm{COCH}_{3}\right), 170.3\left(\mathrm{~s}, \mathrm{COCH}_{3}\right), 169.6\left(\mathrm{~s}, \mathrm{COCH}_{3}\right), 71.9(\mathrm{~d},(\mathrm{C} 3)), 71.2(\mathrm{~d}$, (C4)), 63.2 (t, (C1)), 47.5 (d, (C2)), 39.1 (t), 30.9 (t), 30.0 (t), 29.7 (t), 29.6 $(\mathrm{t}), 29.5(\mathrm{t}), 29.4(\mathrm{t}), 28.0$ (d, (C17)), $27.4(\mathrm{t}), 25.2$ (d), $23.3\left(\mathrm{q}, \mathrm{COCH}_{3}\right)$,

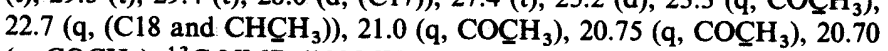
$\left(\mathrm{q}, \mathrm{COCH} \mathrm{CH}_{3}\right){ }^{13} \mathrm{C}-\mathrm{NMR}\left(100 \mathrm{MHz}, \mathrm{C}_{6} \mathrm{D}_{6}\right) \delta: 170.4\left(\mathrm{~s}, \mathrm{COCH}_{3}\right), 170.2(\mathrm{~s}$, $\left.\mathrm{COCH}_{3}\right), 169.1\left(\mathrm{~s}, \mathrm{COCH}_{3}\right), 71.9(\mathrm{~d},(\mathrm{C} 3)), 70.8(\mathrm{~d},(\mathrm{C} 4)), 63.6(\mathrm{t},(\mathrm{C} 1))$, $47.5(\mathrm{~d},(\mathrm{C} 2)), 39.5(\mathrm{t}), 31.6(\mathrm{t}), 30.4(\mathrm{t}), 30.2(\mathrm{t}), 30.1(\mathrm{t}), 30.0(\mathrm{t}), 27.9(\mathrm{t})$, 25.9 (d, (C17)), $22.9\left(\mathrm{q},\left(\mathrm{C} 18, \mathrm{COCH}_{3}\right.\right.$, and $\left.\left.\mathrm{CHCH}_{3}\right)\right), 20.9\left(\mathrm{q}, \mathrm{COCH}_{3}\right)$, $20.4\left(\mathrm{q}, \mathrm{COCH}_{3}\right), 20.2\left(\mathrm{q}, \mathrm{COCH}_{3}\right)$. FD-MS m/z (\%): $500\left(\mathrm{M}^{+}+\mathrm{H}, 100\right)$. Anal. Calcd for $\mathrm{C}_{27} \mathrm{H}_{49} \mathrm{NO}_{7}: \mathrm{C}, 64.90 ; \mathrm{H}, 9.88 ; \mathrm{N}, 2.80$. Found: C, 64.79; $\mathrm{H}, 9.83 ; \mathrm{N}, 2.77$.

(2S,3S,4R,6E)-2-[(R)-2-(Methoxymethoxy)tetracosanoylamino]-17methyl-6-octadecene-1,3,4-triol (28) A solution of DCC (44.8 mg, 217 $\mu \mathrm{mol})$ in THF $(1.5 \mathrm{ml})$ was added to a mixture of $5(47.7 \mathrm{mg}, 145 \mu \mathrm{mol})$, $27(62.2 \mathrm{mg}, 145 \mu \mathrm{mol})$, and HOBT $(33.3 \mathrm{mg}, 217 \mu \mathrm{mol})$. The reaction mixture was stirred at room temperature for $2 \mathrm{~d}$ and filtered through a cotton plug. The filtrate was concentrated and chromatographed on silica gel (BW-300, $\mathrm{CHCl}_{3}$-acetone-water, $\left.85: 15: 0.3\right)$ gave $28(80.9 \mathrm{mg}, 76 \%$ yield) as a colorless powder. $\operatorname{mp} 67.5^{\circ} \mathrm{C}$. $[\alpha]_{\mathrm{D}}^{27}+9.08^{\circ}\left(c=1.96, \mathrm{CHCl}_{3}\right)$. IR $\left(\mathrm{CCl}_{4}\right): 3420(\mathrm{O}-\mathrm{H}$ and $\mathrm{N}-\mathrm{H}), 2930(\mathrm{C}-\mathrm{H}), 2850,1655(\mathrm{NC}=\mathrm{O}) \mathrm{cm}^{-1}$ ${ }^{1} \mathrm{H}$-NMR $\left(270 \mathrm{MHz}^{\left.-\mathrm{CDCl}_{3}\right)} \delta: 7.41(1 \mathrm{H}, \mathrm{d}, J=7.6 \mathrm{~Hz}, \mathrm{NH}), 5.60(1 \mathrm{H}\right.$, $\mathrm{dt}, J=14.6,7.1 \mathrm{~Hz}, 6-\mathrm{H}), 5.41(1 \mathrm{H}, \mathrm{dt}, J=15.0,7.4 \mathrm{~Hz}, 7-\mathrm{H}), 4.69$ $\left(2 \mathrm{H}, \mathrm{s}, \mathrm{OCH}_{2} \mathrm{O}\right), 4.17(1 \mathrm{H}, \mathrm{m}, 2-\mathrm{H}), 4.07\left(1 \mathrm{H}, \mathrm{t}, J=5.6 \mathrm{~Hz}, 2^{\prime}-\mathrm{H}\right), 3.94$ $\left(1 \mathrm{H}, \mathrm{dd}, J=11.9,2.3 \mathrm{~Hz}, 1-\mathrm{H}_{\mathrm{a}}\right), 3.74\left(1 \mathrm{H}, \mathrm{dd}, J=11.9,4.9 \mathrm{~Hz}, 1-\mathrm{H}_{\mathrm{b}}\right), 3.56$ $(2 \mathrm{H}, \mathrm{m}, 3-\mathrm{H}$ and $4-\mathrm{H}), 3.41\left(3 \mathrm{H}, \mathrm{s}, \mathrm{CH}_{3} \mathrm{O}\right), 2.56\left(1 \mathrm{H}, \mathrm{m}, 5-\mathrm{H}_{\mathrm{a}}\right), 2.17(1 \mathrm{H}$, quint., $\left.J=7.5 \mathrm{~Hz}, 5-\mathrm{H}_{\mathrm{b}}\right), 2.03(2 \mathrm{H}, \mathrm{q}, J=6.8 \mathrm{~Hz}, 8-\mathrm{H}), 1.77\left(2 \mathrm{H}, \mathrm{m}, 3^{\prime}-\mathrm{H}\right)$, $1.51(1 \mathrm{H}, \mathrm{dqq}, J=6.6,6.6,6.6 \mathrm{~Hz}, 17-\mathrm{H}), 1.25(\mathrm{~m}), 0.864(3 \mathrm{H}, \mathrm{t}, J=4.1 \mathrm{~Hz}$, 
$\left.24^{\prime}-\mathrm{H}\right), 0.860\left(6 \mathrm{H}, \mathrm{d}, J=6.6 \mathrm{~Hz}, 18-\mathrm{H}\right.$ and $\left.\mathrm{CHCH}_{3}\right)$. FAB-MS $m / z(\%)$ : $740\left(\mathrm{M}^{+}, 14\right), 708\left(\mathrm{M}^{+}-\mathrm{CH}_{3} \mathrm{OH}, 21\right)$. Anal. Calcd for $\mathrm{C}_{45} \mathrm{H}_{89} \mathrm{NO}_{6}: \mathrm{C}$, $73.02 ; \mathrm{H}, 12.12 ; \mathrm{N}, 1.89$. Found: C, 72.77; H, 12.02; N, 1.88

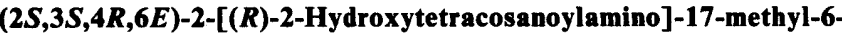
octadecene-1,3,4-triol (3) $\mathrm{BF}_{3} \cdot \mathrm{OEt}_{2}(38.2 \mu \mathrm{l}, 311 \mu \mathrm{mol})$ was added to a solution of $28(76.7 \mathrm{mg}, 104 \mu \mathrm{mol})$ in 1,2-ethanedithiol $(1 \mathrm{ml})$ and $\mathrm{CHCl}_{3}$ $(1 \mathrm{ml})$, and the mixture was stirred at room temperature for $30 \mathrm{~min}$. The reaction was quenched with triethylamine $(144 \mu \mathrm{l}, 1.0 \mathrm{mmol})$, and the mixture was concentrated by blowing a stream of nitrogen gas over it The residue was chromatographed on silica gel (BW-300, $\mathrm{CHCl}_{3}$-acetonewater, $65: 35: 0.7)$ to give $3(54.5 \mathrm{mg}, 76 \%$ yield) as a colorless powder mp 125-126 ${ }^{\circ} \mathrm{C} .[\alpha]_{\mathrm{D}}^{27}+2.89^{\circ}\left(c=1.06, \mathrm{CHCl}_{3}\right)$. IR $\left(\mathrm{CCl}_{4}\right): 3360(\mathrm{O}-\mathrm{H}$ and $\mathrm{N}-\mathrm{H}), 2925(\mathrm{C}-\mathrm{H}), 2850,1642(\mathrm{NC}=\mathrm{O}) \mathrm{cm}^{-1} .{ }^{1} \mathrm{H}-\mathrm{NMR}(270 \mathrm{MHz}$ $\left.\mathrm{C}_{5} \mathrm{D}_{5} \mathrm{~N}\right) \delta: 8.59(1 \mathrm{H}, \mathrm{d}, J=8.9 \mathrm{~Hz}, \mathrm{NH}), 5.99(1 \mathrm{H}, \mathrm{dt}, J=14.8,7.3 \mathrm{~Hz}$ 6-H), $5.74(1 \mathrm{H}, \mathrm{dt}, J=14.7,7.2 \mathrm{~Hz}, 7-\mathrm{H}), 5.13(1 \mathrm{H}, \mathrm{m}, 2-\mathrm{H}), 4.62(1 \mathrm{H}$ dd, $\left.J=7.6,3.6 \mathrm{~Hz}, 2^{\prime}-\mathrm{H}\right), 4.51(1 \mathrm{H}, \mathrm{dd}, J=10.6,4.76 \mathrm{~Hz}, 1-\mathrm{H}), 3.07(1 \mathrm{H}$ $\left.\mathrm{m}, 5-\mathrm{H}_{\mathrm{a}}\right), 2.72\left(1 \mathrm{H}\right.$, quint., $\left.J=7.1 \mathrm{~Hz}, 5-\mathrm{H}_{\mathrm{b}}\right), 2.23\left(1 \mathrm{H}, \mathrm{m}, 3^{\prime}-\mathrm{H}_{\mathrm{a}}\right), 1.50(1 \mathrm{H}$ dqq, $J=6.6,6.6,6.6 \mathrm{~Hz}, 17-\mathrm{H}), 1.32(\mathrm{~m}), 1.26(\mathrm{~m}), 0.88(3 \mathrm{H}, \mathrm{t}, J=6.6 \mathrm{~Hz}$ $\left.24^{\prime}-\mathrm{H}\right), 0.86\left(6 \mathrm{H}, \mathrm{d}, J=6.6 \mathrm{~Hz}, 18-\mathrm{H}\right.$ and $\left.\mathrm{CHCH}_{3}\right){ }^{13} \mathrm{C}-\mathrm{NMR}(67.8 \mathrm{MHz}$, $\left.\mathrm{C}_{5} \mathrm{D}_{5} \mathrm{~N}\right) \delta: 175.3(\mathrm{~s}, \mathrm{CO}), 132.8(\mathrm{~d},(\mathrm{C} 7)), 128.3(\mathrm{~d},(\mathrm{C} 6)), 76.3(\mathrm{~d},(\mathrm{C} 3))$ 73.3 (d, (C4)), 42.5 (d, (C2')), 62.0 (t, (C1)), 52.9 (d, (C2)), 39.3 (t), 37.7 $(\mathrm{t}), 33.2(\mathrm{t},(\mathrm{C})), 32.1(\mathrm{t}), 30.2(\mathrm{t}), 30.0(\mathrm{t}), 29.9(\mathrm{t}), 29.6(\mathrm{t}), 28.2(\mathrm{t}), 27.7$ $(\mathrm{d},(\mathrm{Cl} 17)), 25.8(\mathrm{t}), 22.9(\mathrm{t}), 22.8\left(\mathrm{q},\left(\mathrm{C} 18\right.\right.$ and $\left.\left.\mathrm{CHCH}_{3}\right)\right), 14.3\left(\mathrm{q},\left(\mathrm{C} 24^{\prime}\right)\right)$ FD-MS $m / z(\%): 696\left(\mathrm{M}^{+}+\mathrm{H}, 100\right), 678\left(\mathrm{M}^{+}-\mathrm{H}_{2} \mathrm{O}, 42\right)$. Anal. Calcd for $\mathrm{C}_{43} \mathrm{H}_{85} \mathrm{NO}_{5}$ : C, 74.19; $\mathrm{H}, 12.31 ; \mathrm{N}, 2.01$. Found: $\mathrm{C}, 74.11 ; \mathrm{H}, 12.23$; $\mathrm{N}, 2.05$.

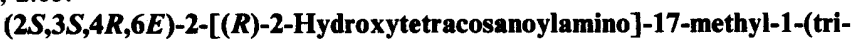
phenylmethyloxy)-6-octadecene-3,4-diol (29) Compound 3 (18.6 mg, 26.7 $\mu \mathrm{mol})$, trityl chloride $(73.2 \mathrm{mg}, 263 \mu \mathrm{mol})$, and dimethylaminopyridine (DMAP) $(21.4 \mathrm{mg}, 175 \mu \mathrm{mol})$ were dissolved in pyridine $(534 \mu \mathrm{l})$. After being stirred at $60^{\circ} \mathrm{C}$ for $4 \mathrm{~h}$, the reaction mixture was diluted with $\mathrm{CHCl}_{3}$, washed with water, dried $\left(\mathrm{Na}_{2} \mathrm{SO}_{4}\right)$, filtered, and concentrated in vacuo. The residue was chromatographed on silica gel $\left(\mathrm{CHCl}_{3}-\mathrm{MeOH}-\mathrm{H}_{2} \mathrm{O}\right.$ $6: 4: 0.8)$ to give $29\left(15.8 \mathrm{mg}, 90 \%\right.$ yield) as a colorless viscous oil. $[\alpha]_{\mathrm{D}}^{25}$ $+16.0^{\circ}\left(c=0.725, \mathrm{CHCl}_{3}\right)$. IR $\left(\mathrm{CCl}_{4}\right): 3410(\mathrm{O}-\mathrm{H}$ and $\mathrm{N}-\mathrm{H}), 3060$ and $3030(\mathrm{Ar}-\mathrm{H}), 2930(\mathrm{C}-\mathrm{H}), 2855,1660(\mathrm{NC}=\mathrm{O}) \mathrm{cm}^{-1} .{ }^{1} \mathrm{H}-\mathrm{NMR}$ $\left(270 \mathrm{MHz}, \mathrm{CDCl}_{3}\right) \delta: 7.41(6 \mathrm{H}, \mathrm{m}$, aromatic $\mathrm{H}), 7.27(9 \mathrm{H}, \mathrm{m}$, aromatic H), $7.12(1 \mathrm{H}, \mathrm{d}, J=8.6 \mathrm{~Hz}, \mathrm{NH}), 5.53(1 \mathrm{H}, \mathrm{dt}, J=14.6,7.1 \mathrm{~Hz}, 6-\mathrm{H}), 5.34$ $(1 \mathrm{H}, \mathrm{dt}, J=14.6,7.3 \mathrm{~Hz}, 7-\mathrm{H}), 4.25(1 \mathrm{H}, \mathrm{m}, 2-\mathrm{H}), 4.07(1 \mathrm{H}, \mathrm{t}, J=5.6 \mathrm{~Hz}$, $\left.2^{\prime}-\mathrm{H}\right), 4.00\left(1 \mathrm{H}, \mathrm{m}, 2^{\prime}-\mathrm{H}\right), 3.62(1 \mathrm{H}, \mathrm{m}, 3-\mathrm{H}), 3.52(1 \mathrm{H}, \mathrm{dd}, J=11.9,2.3 \mathrm{~Hz}$, $\left.1-\mathrm{H}_{\mathrm{s}}\right), 3.43\left(1 \mathrm{H}, \mathrm{dd}, J=9.9,4.0 \mathrm{~Hz}, 1-\mathrm{H}_{\mathrm{b}}\right), 3.26(1 \mathrm{H}, \mathrm{m}, 4-\mathrm{H}), 2.47(1 \mathrm{H}$, $\left.\mathrm{m}, 5-\mathrm{H}_{\mathrm{a}}\right), 2.02(2 \mathrm{H}, \mathrm{m}, 8-\mathrm{H}), 1.26(\mathrm{~m}), 0.86(6 \mathrm{H}, \mathrm{d}, J=6.6 \mathrm{~Hz}, 18-\mathrm{H}$ and $\left.\mathrm{CHCH}_{3}\right)$. Negative FAB-MS $m / z(\%)$ : $936\left(\mathrm{M}^{+}-\mathrm{H}, 4\right), 694\left(\mathrm{M}^{+}-\mathrm{CPh}_{3}\right.$, 19), 424 (41), 153 (100). Anal. Calcd for $\mathrm{C}_{62} \mathrm{H}_{99} \mathrm{NO}_{5}$ : C, 79.35; $\mathrm{H}, 10.63$; $\mathrm{N}, 1.49$. Found: $\mathrm{C}, 79.38 ; \mathrm{H}, 10.57 ; \mathrm{N}, 1.45$.

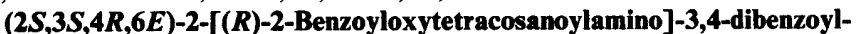
oxy-17-methyl-1-(triphenylmethyloxy)-6-octadecene (30) Benzoyl chloride $(95.0 \mu 1,820 \mu \mathrm{mol})$ was added to a solution of $29(44.2 \mathrm{mg}, 47.1 \mu \mathrm{mol})$ and DMAP $(99.8 \mathrm{mg}, 820 \mu \mathrm{mol})$ in pyridine $(1.6 \mathrm{ml})$. After being stirred at $70-75^{\circ} \mathrm{C}$ for $15 \mathrm{~h}$, the reaction mixture was diluted with water and extracted with $\mathrm{CHCl}_{3}$. The extracts were washed with water, dried $\left(\mathrm{Na}_{2} \mathrm{SO}_{4}\right)$, filtered, and concentrated in vacuo. The residue was chromatographed on silica gel (hexane-AcOEt, $3: 1)$ to give $30(58.0 \mathrm{mg}, 98 \%$ yield) as a colorless viscous oil. $[\alpha]_{\mathrm{D}}^{29}+30.9^{\circ}\left(c=2.43, \mathrm{CHCl}_{3}\right)$. IR $\left(\mathrm{CCl}_{4}\right): 3060$ and $3030(\mathrm{Ar}-\mathrm{H}), 2920(\mathrm{C}-\mathrm{H}), 2850,1725(\mathrm{OC}=\mathrm{O}), 1682(\mathrm{NC}=\mathrm{O}) \mathrm{cm}^{-1}$. ${ }^{1} \mathrm{H}$-NMR $\left(270 \mathrm{MHz}, \mathrm{CDCl}_{3}\right) \delta: 8.05(2 \mathrm{H}, \mathrm{d}, J=7.6 \mathrm{~Hz}$, aromatic $\mathrm{H}), 7.96$ $(2 \mathrm{H}, \mathrm{d}, J=7.6 \mathrm{~Hz}$, aromatic $\mathrm{H}), 7.86(2 \mathrm{H}, \mathrm{d}, J=7.6 \mathrm{~Hz}$, aromatic $\mathrm{H}), 7.52$ $(3 \mathrm{H}, \mathrm{m}$, aromatic $\mathrm{H}), 7.39(4 \mathrm{H}, \mathrm{t}, J=7.6 \mathrm{~Hz}$, aromatic $\mathrm{H}), 7.27(2 \mathrm{H}, \mathrm{t}$, $J=7.8 \mathrm{~Hz}$, aromatic $\mathrm{H}), 7.12(6 \mathrm{H}, \mathrm{m}$, aromatic $\mathrm{H}), 7.00(9 \mathrm{H}, \mathrm{m}$, aromatic H), $5.85(1 \mathrm{H}$, dd, $J=8.2,3.3 \mathrm{~Hz}, 3-\mathrm{H}), 5.57\left(1 \mathrm{H}, \mathrm{t}, J=5.8 \mathrm{~Hz}, 2^{\prime}-\mathrm{H}\right), 5.53$ (1H, m, 6-H), 5.39. (1H, m, 4-H), $5.34(1 \mathrm{H}, \mathrm{m}, 7-\mathrm{H}), 4.64(1 \mathrm{H}, \mathrm{m}, 2-\mathrm{H})$, $3.24(\mathrm{~d}, J=3.3 \mathrm{~Hz}, 2 \mathrm{H}, 1-\mathrm{H}), 2.66\left(\mathrm{~m}, 1 \mathrm{H}, 5-\mathrm{H}_{\mathrm{a}}\right), 2.49\left(1 \mathrm{H}, \mathrm{m}, 5-\mathrm{H}_{\mathrm{b}}\right), 2.06$ $\left(2 \mathrm{H}, \mathrm{m}, 3^{\prime}-\mathrm{H}\right), 1.87(2 \mathrm{H}, \mathrm{m}, 8-\mathrm{H}), 1.26(\mathrm{~m}), 0.88(3 \mathrm{H}, \mathrm{t}, J=6.3 \mathrm{~Hz}$, $\left.\mathrm{CH}_{2} \mathrm{CH}_{3}\right), 0.85\left(6 \mathrm{H}, \mathrm{d}, J=6.6 \mathrm{~Hz}, 18-\mathrm{H}\right.$ and $\left.\mathrm{CHCH}_{3}\right)$. Negative FAB-MS $m / z(\%): 1248\left(\mathrm{M}^{+}-\mathrm{H}, 1.2\right), 486(1.1)$. Anal. Calcd for $\mathrm{C}_{83} \mathrm{H}_{111} \mathrm{NO}_{8}: \mathrm{C}$, 79.70; H, 8.94; N, 1.12. Found: C, 79.83; H, 8.94; N, 1.12 .

$(2 S, 3 S, 4 R, 6 E)-2-[(R)-2$-Benzoyloxytetracosanoylamino]-3,4-dibenzoyloxy-17-methyl-6-octadecen-1-ol (31) A solution of $\mathrm{TsOH} \cdot \mathrm{H}_{2} \mathrm{O}(17.7 \mathrm{mg}$, $93 \mu \mathrm{mol})$ in $\mathrm{CHCl}_{3}(1.25 \mathrm{ml})$ and $\mathrm{MeOH}(0.6 \mathrm{ml})$ was added to $30(58.0 \mathrm{mg}$, $46.4 \mu \mathrm{mol}$ ). After being stirred at room temperature for $8 \mathrm{~h}$, the mixture was concentrated to $1 / 4$ of the original volume by blowing a stream of nitrogen gas over it, and the concentrate was chromatographed on silica gel (hexane-AcOEt, 7:3) to give $31(44.3 \mathrm{mg}, 95 \%$ yield) as a colorless viscous oil. $[\alpha]_{\mathrm{D}}^{26}+43.7^{\circ}\left(c=1.88, \mathrm{CHCl}_{3}\right)$. IR $\left(\mathrm{CCl}_{4}\right): 3520$ and 3420 $(\mathrm{O}-\mathrm{H}$ and $\mathrm{N}-\mathrm{H}), 3060$ and $3030(\mathrm{Ar}-\mathrm{H}), 2930(\mathrm{C}-\mathrm{H}), 2850,1725(\mathrm{OC}=\mathrm{O})$,
$1685(\mathrm{NC}=\mathrm{O}) \mathrm{cm}^{-1} .{ }^{1} \mathrm{H}-\mathrm{NMR}\left(270 \mathrm{MHz}, \mathrm{CDCl}_{3}\right) \delta: 8.14(2 \mathrm{H}, \mathrm{d}$, $J=7.9 \mathrm{~Hz}$, aromatic $\mathrm{H}), 8.01(2 \mathrm{H}, \mathrm{d}, J=8.2 \mathrm{~Hz}$, aromatic $\mathrm{H}), 7.96(2 \mathrm{H}$, $\mathrm{d}, J=8.4 \mathrm{~Hz}$, aromatic $\mathrm{H}), 7.45(9 \mathrm{H}, \mathrm{m}$, aromatic $\mathrm{H}), 7.13(1 \mathrm{H}, \mathrm{d}$, $J=9.2 \mathrm{~Hz}, \mathrm{NH}), 5.65-5.35\left(5 \mathrm{H}, \mathrm{m}, 3-\mathrm{H}, 4-\mathrm{H}, 6-\mathrm{H}, 7-\mathrm{H}\right.$, and $\left.2^{\prime}-\mathrm{H}\right), 4.44$ $(1 \mathrm{H}, \mathrm{m}, 2-\mathrm{H}), 3.64(2 \mathrm{H}, \mathrm{br} \mathrm{s}, 1-\mathrm{H}), 2.78\left(1 \mathrm{H}, \mathrm{m}, 5-\mathrm{H}_{\mathrm{a}}\right), 2.65\left(1 \mathrm{H}, \mathrm{m}, 5-\mathrm{H}_{\mathrm{b}}\right)$, $2.07(2 \mathrm{H}, \mathrm{m}), 2.92(2 \mathrm{H}, \mathrm{m}), 1.26(\mathrm{~m}), 0.88\left(3 \mathrm{H}, \mathrm{t}, J=6.6 \mathrm{~Hz}, \mathrm{CH}_{2} \mathrm{CH}_{3}\right)$, $0.86\left(6 \mathrm{H}, \mathrm{d}, J=6.6 \mathrm{~Hz}, 18-\mathrm{H}\right.$ and $\left.\mathrm{CHCH}_{3}\right)$. FAB-MS $m / z(\%): 1008$ $\left(\mathrm{M}^{+}+\mathrm{H}, 11\right), 990\left(\mathrm{M}^{+}-\mathrm{OH}, 16\right), 886\left(\mathrm{M}^{+}-\mathrm{PhCOO}, 100\right)$. Anal. Calcd for $\mathrm{C}_{64} \mathrm{H}_{97} \mathrm{NO}_{8}: \mathrm{C}, 76.22 ; \mathrm{H}, 9.70 ; \mathrm{N}, 1.39$. Found: $\mathrm{C}, 75.97 ; \mathrm{H}, 9.67 ; \mathrm{N}$, 1.42 .

$1-O-(2,3,4,6$-Tetra- $O$-acetoxy- $\beta$-D-galactopyranosyloxy)-(2S,3S,4R,6E)2-[(R)-2-benzoyloxytetracosanoylamino]-3,4-dibenzoyloxy-17-methyl-6octadecene (33) and $(2 S, 3 S, 4 R, 6 E)-1-A c e t o x y-2-[(R)-2$-benzoyloxytetracosanoylamino]-3,4-dibenzyloxy-17-methyl-6-octadecane (34) A suspension of $31(15.9 \mathrm{mg}, 15.8 \mu \mathrm{mol}), 32(25.3 \mathrm{mg}, 53.1 \mu \mathrm{mol})$, and $4 \AA$ molecular sieves $(38.9 \mathrm{mg})$ in $\mathrm{CH}_{2} \mathrm{Cl}_{2}(315 \mu \mathrm{l})$ was stirred at room temperature for $1 \mathrm{~h}$ and boron trifluoride etherate $(5.82 \mu \mathrm{l}, 47.3 \mu \mathrm{mol})$ was added at $-25^{\circ} \mathrm{C}$. After being stirred at $-25^{\circ} \mathrm{C}$ for $1 \mathrm{~h}$, the reaction mixture was allowed to warm to room temperature over $1 \mathrm{~h}$ and stirred at ambient temperature for $1 \mathrm{~h}$. The mixture was diluted with $\mathrm{CHCl}_{3}$, washed with aqueous saturated $\mathrm{NaHCO}_{3}$, dried $\left(\mathrm{Na}_{2} \mathrm{SO}_{4}\right)$, filtered, and concentrated in vacuo. The residue was chromatographed on silica gel (hexane-AcOEt, $8: 2-7: 3)$ to give $33(15.7 \mathrm{mg}, 74 \%$ yield) and $34(4.2 \mathrm{mg}, 25 \%$ yield $)$.

33: $[\alpha]_{\mathrm{b}}^{27}+24^{\circ}\left(c=0.82, \mathrm{CHCl}_{3}\right)$. IR $\left(\mathrm{CCl}_{4}\right): 2930(\mathrm{C}-\mathrm{H}), 2850,1755$ and $1725(\mathrm{OC}=\mathrm{O}), 1685(\mathrm{NC}=\mathrm{O}) \mathrm{cm}^{-1} \cdot{ }^{1} \mathrm{H}-\mathrm{NMR}\left(270 \mathrm{MHz}, \mathrm{CDCl}_{3}\right)$ $\delta: 8.16(2 \mathrm{H}$, dd, $J=7.8,1.7 \mathrm{~Hz}$, aromatic $\mathrm{H}), 8.00(2 \mathrm{H}, \mathrm{dd}, J=5.9,1.3 \mathrm{~Hz}$, aromatic $\mathrm{H}), 7.97(2 \mathrm{H}, \mathrm{dd}, J=9.8,1.8 \mathrm{~Hz}$, aromatic $\mathrm{H}), 7.60(3 \mathrm{H}, \mathrm{m}$, aromatic $\mathrm{H}), 7.45(6 \mathrm{H}, \mathrm{m}$, aromatic $\mathrm{H}), 6.07(1 \mathrm{H}, \mathrm{d}, J=8.9 \mathrm{~Hz}, \mathrm{NH}), 5.60$ $(1 \mathrm{H}, \mathrm{dd}, J=7.89,4.0 \mathrm{~Hz}, 6-\mathrm{H}), 4.63(1 \mathrm{H}, \mathrm{m}, 2-\mathrm{H}), 4.38(1 \mathrm{H}, \mathrm{d}, J=7.9 \mathrm{~Hz}$, $\left.1^{\prime \prime}-\mathrm{H}\right), 2.07(3 \mathrm{H}, \mathrm{s}, \mathrm{Ac}), 1.96(3 \mathrm{H}, \mathrm{s}, \mathrm{Ac}), 1.91(3 \mathrm{H}, \mathrm{s}, \mathrm{Ac}), 1.71(3 \mathrm{H}, \mathrm{s}$, Ac), $1.25(\mathrm{~m}), 0.88\left(3 \mathrm{H}, \mathrm{t}, J=6.8 \mathrm{~Hz}, \mathrm{CH}_{2} \mathrm{CH}_{3}\right), 0.85(6 \mathrm{H}, \mathrm{d}, J=6.6 \mathrm{~Hz}$, $18-\mathrm{H}$ and $\left.\mathrm{CHCH}_{3}\right)$. FAB-MS $m / z(\%)$ : $1338\left(\mathrm{M}^{+}+\mathrm{H}, 2.0\right), 1216$ $\left(\mathrm{M}^{+}-\mathrm{PhCOO}, 3.5\right), 990\left(\mathrm{M}^{+}-\right.$tetraacetylgalactose unit, 8.4). This product was used in the next reaction without further purification.

34: $[\alpha]_{\mathrm{D}}^{25}+17^{\circ}\left(c=0.21, \mathrm{CHCl}_{3}\right)$. IR $\left(\mathrm{CCl}_{4}\right): 2925(\mathrm{C}-\mathrm{H}), 2850,1730$ $(\mathrm{OC}=\mathrm{O}), 1690(\mathrm{NC}=\mathrm{O}) \mathrm{cm}^{-1} .{ }^{1} \mathrm{H}-\mathrm{NMR}\left(270 \mathrm{MHz}, \mathrm{CDCl}_{3}\right) \delta: 8.10(2 \mathrm{H}$, dd, $J=8.3,1.3 \mathrm{~Hz}$, aromatic $\mathrm{H}), 8.00(2 \mathrm{H}, \mathrm{dd}, J=8.4,1.5 \mathrm{~Hz}$, aromatic $\mathrm{H}), 7.96(2 \mathrm{H}, \mathrm{dd}, J=8.9,1.5 \mathrm{~Hz}$, aromatic $\mathrm{H}), 7.59(3 \mathrm{H}, \mathrm{m}$, aromatic $\mathrm{H})$, $7.43(6 \mathrm{H}, \mathrm{m}$, aromatic $\mathrm{H}), 6.92(1 \mathrm{H}, \mathrm{d}, J=9.2 \mathrm{~Hz}, \mathrm{NH}), 5.68-5.30(5 \mathrm{H}$, $\mathrm{m}, 3-\mathrm{H}, 4-\mathrm{H}, 6-\mathrm{H}, 7-\mathrm{H}$, and $\left.2^{\prime}-\mathrm{H}\right), 4.77(1 \mathrm{H}, \mathrm{m}, 2-\mathrm{H}), 4.24(1 \mathrm{H}, \mathrm{dd}, J=11.7$, $\left.5.1 \mathrm{~Hz}, 1-\mathrm{H}_{\mathrm{a}}\right), 4.16\left(1 \mathrm{H}, \mathrm{dd}, J=11.7,4.1 \mathrm{~Hz}, 1-\mathrm{H}_{\mathrm{b}}\right), 2.06(2 \mathrm{H}, \mathrm{m}), 1.88(2 \mathrm{H}$, m), $1.70(3 \mathrm{H}, \mathrm{s}, \mathrm{Ac}), 1.25(\mathrm{~m}), 0.87\left(3 \mathrm{H}, \mathrm{t}, J=7.3 \mathrm{~Hz}, \mathrm{CH}_{2} \mathrm{CH}_{3}\right), 0.86(6 \mathrm{H}$, d, $J=6.6 \mathrm{~Hz}, 18-\mathrm{H}$ and $\left.\mathrm{CHCH}_{3}\right)$. FD-MS $m / z(\%): 1050\left(\mathrm{M}^{+}+\mathrm{H}, 100\right)$ Anal. Calcd for $\mathrm{C}_{66} \mathrm{H}_{99} \mathrm{NO}_{9}: \mathrm{C}, 75.46 ; \mathrm{H}, 9.50 ; \mathrm{N}, 1.33$. Found: $\mathrm{C}, 75.27$; $\mathrm{H}, 9.46 ; \mathrm{N}, 1.29$.

1-O-( $\beta$-D-Galactopyranosyloxy)-(2S,3S,4R,6E)-2-[(R)-2-hydroxytetracosanoylamino]-17-methyl-6-octadecene-3,4-diol (2) A $0.1 \mathrm{M}$ solution of $\mathrm{K}_{2} \mathrm{CO}_{3}$ in $\mathrm{MeOH}(1.2 \mathrm{ml})$ was added to a solution of $33(15.7 \mathrm{mg})$ in $\mathrm{MeOH}(0.6 \mathrm{ml})$. After being stirred at room temperature for $30 \mathrm{~min}$, the reaction mixture was concentrated by blowing a stream of nitrogen gas over it, and the residue was chromatographed on silica gel (BW-300, $\left.\mathrm{CHCl}_{3}-\mathrm{MeOH}-\mathrm{H}_{2} \mathrm{O}, 85: 15: 1.5\right)$ to give $2(8.7 \mathrm{mg}, 89 \%$ yield $)$ as colorless crystals. mp $218-220^{\circ} \mathrm{C}$. $[\alpha]_{\mathrm{D}}^{27}+18^{\circ}\left(c=0.34, \mathrm{CHCl}_{3}-\mathrm{MeOH}, 2: 1\right)$. IR $(\mathrm{KBr}): 3400(\mathrm{O}-\mathrm{H}), 2920(\mathrm{C}-\mathrm{H}), 2850(\mathrm{C}-\mathrm{H}), 1645(\mathrm{NC}=\mathrm{O}), 1540,1075$, $720 \mathrm{~cm}^{-1} .{ }^{1} \mathrm{H}-\mathrm{NMR}\left(270 \mathrm{MHz}, \mathrm{C}_{5} \mathrm{D}_{5} \mathrm{~N}\right) \delta: 8.57(1 \mathrm{H}, \mathrm{d}, J=9.6 \mathrm{~Hz}, \mathrm{NH})$, $5.99(1 \mathrm{H}, \mathrm{dt}, J=14.6,7.2 \mathrm{~Hz}, 6-\mathrm{H}), 5.74(1 \mathrm{H}, \mathrm{dt}, J=14.5,7.1 \mathrm{~Hz}, 7-\mathrm{H})$, $5.29(1 \mathrm{H}, \mathrm{m}, 2-\mathrm{H}), 4.89(1 \mathrm{H}, \mathrm{d}, J=7.9 \mathrm{~Hz}), 4.78(1 \mathrm{H}, \mathrm{dd}, J=10.6,6.6 \mathrm{~Hz})$, $4.13(3 \mathrm{H}, \mathrm{dd}, J=9.2,3.3 \mathrm{~Hz}), 4.02(1 \mathrm{H}, \mathrm{t}, J=5.9 \mathrm{~Hz}), 3.04(1 \mathrm{H}$, dd, $\left.J=12.5,6.6 \mathrm{~Hz}, 5-\mathrm{H}_{\mathrm{a}}\right), 2.70\left(1 \mathrm{H}\right.$, quint., $\left.J=7.2 \mathrm{~Hz}, 5-\mathrm{H}_{\mathrm{b}}\right), 1.50(1 \mathrm{H}$, dqq, $J=6.5,6.6,6.6 \mathrm{~Hz}, 17-\mathrm{H}), 1.33(\mathrm{~m}), 1.27(\mathrm{~m}), 0.879(6 \mathrm{H}, \mathrm{d}, J=6.6 \mathrm{~Hz}$, $18-\mathrm{H}$ and $\left.\mathrm{CHCH}_{3}\right), 0.877\left(3 \mathrm{H}, \mathrm{t}, J=6.9 \mathrm{~Hz}, 24^{\prime}-\mathrm{H}\right) .{ }^{13} \mathrm{C}-\mathrm{NMR}(67.8 \mathrm{MHz}$, $\left.\mathrm{C}_{5} \mathrm{D}_{5} \mathrm{~N}\right) \delta: 175.7$ (s), 132.7 (d), 128.3 (d), 106.3 (d), 77.1 (d), 75.3 (d), 75.2 (d), 72.8 (d), 72.6 (d), 72.4 (d), 70.6 (t), 70.2 (d), 62.4 (t), 51.6 (d), 39.3 $(\mathrm{t}), 37.5(\mathrm{t}), 35.6(\mathrm{t}), 33.3(\mathrm{t}), 32.1(\mathrm{t}), 30.3(\mathrm{t}), 30.1(\mathrm{t}), 29.9(\mathrm{t}), 29.73(\mathrm{t})$, $29.65(\mathrm{t}), 29.6(\mathrm{t}), 28.2(\mathrm{~d}), 27.7(\mathrm{t}), 25.9(\mathrm{t}), 22.9(\mathrm{t}), 22.8(\mathrm{q}), 14.3(\mathrm{q})$. FD-MS $m / z(\%): 858\left(\mathbf{M}^{+}+\mathrm{H}, 100\right), 840\left(\mathrm{M}^{+}-\mathrm{OH}, 27\right)$. Anal. Calcd for $\mathrm{C}_{64} \mathrm{H}_{97} \mathrm{NO}_{8} \cdot 0.5 \mathrm{H}_{2} \mathrm{O}: \mathrm{C}, 67.86 ; \mathrm{H}, 11.16 ; \mathrm{N}, 1.61$. Found: $\mathrm{C}, 67.79 ; \mathrm{H}$, $10.97 ; \mathrm{N}, 1.60$. [Spectral data of the mixture of $1 \mathrm{a}$ and $1 \mathrm{~b}^{2)}$ IR $(\mathrm{KBr})$ : 3361,1076 , and $1047(\mathrm{O}-\mathrm{H}), 1656$ and $1544(\mathrm{NC}=\mathrm{O}) \mathrm{cm}^{-1} \cdot{ }^{1} \mathrm{H}-\mathrm{NMR}$ $\left(270 \mathrm{MHz}, \mathrm{C}_{5} \mathrm{D}_{5} \mathrm{~N}\right) \delta: 5.94(\mathrm{dt}, J=15.4,8.1 \mathrm{~Hz}), 5.71(\mathrm{dt}, J=15.4,8.1 \mathrm{~Hz})$, 4.0-4.7 (sugar protons), 4.87 (d, $J=7.5 \mathrm{~Hz}$, anomeric proton), 1.25 (long chain methylene protons), $0.85(6 \mathrm{H}, \mathrm{d}, J=7.3 \mathrm{~Hz}$, secondary methyls), $0.85\left(3 \mathrm{H}, \mathrm{t}, J=7.0 \mathrm{~Hz}\right.$, primary methyl). ${ }^{13} \mathrm{C}-\mathrm{NMR}\left(67 \mathrm{MHz}, \mathrm{C}_{5} \mathrm{D}_{5} \mathrm{~N}\right) \delta$ : 175.61 (carbonyl), 133.67 and 126.60 (olefinic carbons)]. The ${ }^{1} \mathrm{H}-\mathrm{NMR}$ spectrum of 2 was superimposable on that of the mixture of $1 \mathbf{a}$ and $\mathbf{1 b}$ 
provided by Dr. M. Nakagawa.

1-O-(2,3,4,6-Tetra- $O$-acetoxy- $\beta$-D-galactopyranosyloxy)-(2S,3S,4R,6E)2-[(R)-2-acetoxytetracosanoylamino]-3,4-diacetoxy-17-methyl-6-octadecene (35) Acetic anhydride $(175 \mu \mathrm{l})$ and pyridine $(151 \mu \mathrm{l})$ were added to 2 $(8.0 \mathrm{mg})$. After being stirred at $50^{\circ} \mathrm{C}$ for $3 \mathrm{~h}$, the reaction mixture was concentrated by blowing a stream of nitrogen gas over it and the residue was chromatographed on silica gel (BW-300, hexane-AcOEt, 6:4) to give $35(9.5 \mathrm{mg}, 88 \%$ yield $)$ as a colorless viscous oil. $[\alpha]_{\mathrm{D}}^{26}+2.8^{\circ}(c=0.44$ $\left.\mathrm{CHCl}_{3}\right)$. IR $\left(\mathrm{CCl}_{4}\right): 2925(\mathrm{C}-\mathrm{H}), 2850(\mathrm{C}-\mathrm{H}), 1755(\mathrm{OC}=\mathrm{O}), 1685$ $(\mathrm{NC}=\mathrm{O}) \mathrm{cm}^{-1} .{ }^{1} \mathrm{H}-\mathrm{NMR}\left(270 \mathrm{MHz}, \mathrm{CDCl}_{3}\right) \delta: 6.78(1 \mathrm{H}, \mathrm{d}, J=8.9 \mathrm{~Hz}$, $\mathrm{NH}), 5.48(1 \mathrm{H}, \mathrm{dt}, J=14.6,7.2 \mathrm{~Hz}, 6-\mathrm{H}), 5.37\left(1 \mathrm{H}, \mathrm{d}, J=2.8 \mathrm{~Hz}, 4^{\prime \prime}-\mathrm{H}\right)$, $5.26(1 \mathrm{H}, \mathrm{dt}, J=14.7,7.3 \mathrm{~Hz}, 7-\mathrm{H}), 5.19-5.08\left(2 \mathrm{H}, \mathrm{m}, 3-\mathrm{H}\right.$, and $\left.2^{\prime}-\mathrm{H}\right)$ $5.00\left(1 \mathrm{H}, \mathrm{dd}, J=10.6,3.3 \mathrm{~Hz}, 3^{\prime \prime}-\mathrm{H}\right), 4.92(1 \mathrm{H}, \mathrm{dt}, J=9.1,3.7 \mathrm{~Hz}, 4-\mathrm{H})$ $4.45\left(1 \mathrm{H}, \mathrm{d}, J=7.6 \mathrm{~Hz}, \mathrm{l}^{\prime \prime}-\mathrm{H}\right), 4.33(1 \mathrm{H}, \mathrm{m}, 2-\mathrm{H}), 4.13(2 \mathrm{H}, \mathrm{d}, J=6.6 \mathrm{~Hz}$ $\left.6^{\prime \prime}-\mathrm{H}\right), 3.90\left(1 \mathrm{H}, \mathrm{dd}, J=7.6,6.6 \mathrm{~Hz}, 5^{\prime \prime}-\mathrm{H}\right), 3.86(1 \mathrm{H}, \mathrm{dd}, J=10.9,3.6 \mathrm{~Hz}$ $\left.1-\mathrm{H}_{\mathrm{a}}\right), 3.68\left(1 \mathrm{H}, \mathrm{dd}, J=10.9,3.6 \mathrm{~Hz}, 1-\mathrm{H}_{\mathrm{b}}\right), 2.23\left(3 \mathrm{H}, \mathrm{s}, \mathrm{CH}_{3} \mathrm{CO}\right), 2.15$ $\left(3 \mathrm{H}, \mathrm{s}, \mathrm{CH}_{3} \mathrm{CO}\right), 2.10\left(3 \mathrm{H}, \mathrm{s}, \mathrm{CH}_{3} \mathrm{CO}\right), 2.05\left(3 \mathrm{H}, \mathrm{s}, \mathrm{CH}_{3} \mathrm{CO}\right), 2.04(3 \mathrm{H}$ s, $\left.\mathrm{CH}_{3} \mathrm{CO}\right), 1.97\left(3 \mathrm{H}, \mathrm{s}, \mathrm{CH}_{3} \mathrm{CO}\right), 1.25(\mathrm{~m}), 0.88(3 \mathrm{H}, \mathrm{t}, J=6.6 \mathrm{~Hz}$, $\left.\mathrm{CH}_{2} \mathrm{CH}_{3}\right), 0.86\left(6 \mathrm{H}, \mathrm{d}, J=6.6 \mathrm{~Hz}, 18-\mathrm{H}\right.$ and $\left.\mathrm{CHCH}_{3}\right) .{ }^{13} \mathrm{C}-\mathrm{NMR}$ $\left(67.8 \mathrm{MHz}, \mathrm{CDCl}_{3}\right.$ ) $\delta: 170.7$ (s), 170.3 (s), 170.2 (s), 170.1 (s), 170.05 (s) 169.99 (s), 169.8 (s), 169.4 (s), 134.6 (d), 124.0 (d), 100.7 (d), 73.9 (d), 72.6 (d), 72.0 (d), 70.8 (d), 68.6 (d), 67.0 (d), 66.2 (t), 61.1 (t), 48.1 (d), 39.1 $(\mathrm{t}), 32.6(\mathrm{t}), 32.1(\mathrm{t}), 31.9(\mathrm{t}), 31.8(\mathrm{t}), 30.0(\mathrm{t}), 29.7(\mathrm{t}), 29.6(\mathrm{t}), 29.5(\mathrm{t})$, $29.4(\mathrm{t}), 29.3(\mathrm{t}), 29.2(\mathrm{t}), 28.0(\mathrm{~d}), 27.4(\mathrm{t}), 24.9(\mathrm{t}), 22.7$ (q), $20.99(\mathrm{q})$, 20.95 (q), 20.7 (q), 20.6 (q), 20.5 (q), 14.1 (q). FAB-MS m/z (\%): 1152 $\left(\mathrm{M}^{+}+\mathrm{H}, 1.2\right), 1092\left(\mathrm{M}^{+}-\mathrm{AcOH}, 1.5\right), 804\left(\mathrm{M}^{+}\right.$- tetraacetylgalactose unit, 8.9). Anal. Calcd for $\mathrm{C}_{64} \mathrm{H}_{97} \mathrm{NO}_{8}: \mathrm{C}, 65.65 ; \mathrm{H}, 9.53 ; \mathrm{N}, 1.22$. Found: C, 65.38; H, 9.50; N, 1.24. [NMR data of the mixture of acetates of 1a and $1 \mathrm{~b}^{16)}{ }^{1} \mathrm{H}-\mathrm{NMR}\left(360 \mathrm{MHz}, \mathrm{CDCl}_{3}\right) \delta: 4.44\left(1 \mathrm{H}, \mathrm{d}, J=7.5 \mathrm{~Hz}, 1^{\prime \prime}-\mathrm{H}\right)$, $3.86\left(1 \mathrm{H}, \mathrm{dd}, J=10.8,3.0 \mathrm{~Hz}, 1-\mathrm{H}_{\mathrm{a}}\right), 3.67\left(1 \mathrm{H}, \mathrm{dd}, J=10.8,3.5 \mathrm{~Hz}, 1-\mathrm{H}_{\mathrm{b}}\right)$. ${ }^{13} \mathrm{C}-\mathrm{NMR}\left(67 \mathrm{MHz}, \mathrm{CDCl}_{3}\right.$ ) $\delta: 134.3$ (d), 123.9 (d), 100.6 (d), 73.9 (d), 72.6 (d), 72.1 (d), 70.9 (d), 68.7 (d), 67.0 (d), 66.3 (t), 61.1 (t), 48.2 (d), 39.2 (t), 28.2 (d), 25.1 (t), 22.8 (q), 14.3 (q)]. The ${ }^{13} \mathrm{C}$ - and ${ }^{1} \mathrm{H}-\mathrm{NMR}$ spectral data of 35 were superimposable on those of the mixture of acetates of $1 \mathrm{a}$ and $\mathbf{1 b}$ provided by Dr. M. Nakagawa.

Acknowledgements We are grateful to Dr. $M$. Endo and Dr. $M$ Nakagawa of Suntory Institute for Biological Research for providing the NMR data on the cerebrosides. Thanks are also due to Mr. A. Tanaka, Miss Y. Soeda, and Mr. R. Isobe of the Faculty of Pharmaceutical Sciences,
Kyushu University, for recording NMR and mass spectra. This work was supported in part by a Grant-in-Aid for Scientific Research (No. 01771919) from the Ministry of Education, Science, and Culture of Japan.

\section{References and Notes}

1) Some of these data were presented at the 31st Symposium on the Chemistry of Natural Products, Nagoya, Japan, Oct. 1989, Symposium Papers, p. 22.

2) M. Endo, M. Nakagawa, Y. Hamamoto, and M. Ishihama, Pure Appl. Chem., 58, 387 (1986).

3) a) K. Mori and T. Kinsho, Justus Liebigs Ann. Chem., 1988, 807; b) T. Bär and R. R. Schmidt, ibid., 1990, 669; c) S. Kodato, M. Nakagawa, K. Nakayama, and T. Hino, Tetrahedron, 45, 7247 (1989); d) S. Kodato, M. Nakagawa, and T. Hino, ibid., 45, 7263 (1989); e) M. Nakagawa, S. Kodato, K. Nakayama, and T. Hino, Tetrahedron Lett., 28, 6281 (1987); $f$ ) K. Mori and Y. Funami, Tetrahedron, 41, 2379 (1985).

4) a) S. Sugiyama, M. Honda, and T. Komori, Justus Liebigs Ann. Chem., 1990, 1069; b) Idem, ibid., 1988, 619.

5) E. J. Corey and P. L. Fuchs, Tetrahedron Lett., 1972, 3769.

6) C. A. Brown and A. Yamashita, J. Am. Chem. Soc., 97, 891 (1975).

7) J. Jurczak, S. Pikul, and T. Bauer, Tetrahedron, 42, 447 (1986).

8) A. J. Mancuso, S. L. Huang, and D. Swern, J. Org. Chem., 43, 2480 (1978).

9) a) R. M. Hanson and K. B. Sharpless, J. Org. Chem., 51, 1922 (1986); b) T. Katsuki and K. B. Sharpless, J. Am. Chem. Soc., 102, 5974 (1980).

10) J. M. Finan and Y. Kishi, Tetrahedron Lett., 23, 2719 (1982).

11) S. Tanaka, H. Yamamoto, H. Nozaki, K. B. Sharpless, R. C. Michaelson, and J. D. Cutting, J. Am. Chem. Soc., 96, 5254 (1974).

12) W. R. Roush and M. A. Adam, J. Org. Chem., 50, 3752 (1985).

13) a) W. König and R. Geiger, Chem. Ber., 103, 788 (1970); b) Idem, ibid., 103, 2024 (1970); c) Idem, ibid., 103, 2034 (1970).

14) M. Node, H. Hori, and E. Fujita, J. Chem. Soc., Perkin Trans. 1, 1976, 2237.

15) R. R. Schmidt, Angew. Chem. Int. Ed. Engl., 25, 212 (1986).

16) Unpublished data of M. Endo, M. Nakagawa, Y. Hamamoto, and M. Ishihama. ${ }^{2)}$ 